\title{
Equal-Time Hierarchies for Quantum Transport Theory
}

\author{
Pengfei Zhuang* \\ Gesellschaft für Schwerionenforschung, Theory Group, \\ P.O.Box 110552, D-64220 Darmstadt, Germany \\ Ulrich Heinz \\ Institut für Theoretische Physik, Universität Regensburg, \\ D-93040 Regensburg, Germany
}

(August 10, 2021)

\begin{abstract}
We investigate in the equal-time formalism the derivation and truncation of infinite hierarchies of equations of motion for the energy moments of the covariant Wigner function. From these hierarchies we then extract kinetic equations for the physical distribution functions which are related to loworder energy moments, and show how to determine the higher order moments in terms of these lowest order ones. We apply the general formalism to scalar and spinor QED with classical background fields and compare with the results derived from the three-dimensional Wigner transformation method.
\end{abstract}

PACS: 03.65.Bz, 05.60.+w, 52.60.+h. 


\section{INTRODUCTION}

Transport theory [1] based on the Wigner operator is extensively used to describe the formation and evolution of highly excited nuclear matter produced in relativistic heavy ion collisions. The Wigner operator can be defined in 4-dimensional [2] 6] or 3-dimensional [7],8] momentum space, which we denote by $\hat{\mathcal{W}}(x, p)$ and $\hat{W}(x, \boldsymbol{p})$, respectively. Correspondingly, there are two formulations for the phase-space structure of any field. Either of these two formulations has its advantages and disadvantages. Besides its manifest Lorentz covariance (which is very useful from a technical point of view), another characteristic feature of the 4-dimensional formulation for QCD [2-5] and QED [6] is that the quadratic kinetic equation can be split up naturally into a transport and a constraint equation. The complementarity of these two ingredients is essential for a physical understanding of quantum kinetic theory [9]. In the classical limit, these two equations reduce to the Vlasov and mass-shell equations, respectively. The main advantage of the 3-dimensional approach [7], 8] is that it is easier to set up as an initial value problem: one can directly compute the initial value of the Wigner operator from the corresponding field operators at the same time. In the covariant frame this is not possible since the covariant Wigner operator is defined as a 4-dimensional Wigner transform of the density matrix and thus includes an integration over time. Hence in this approach the initial condition for the Wigner operator at very early times must be constructed phenomenologically. Some true quantum problems like pair production 10] in a strong external field have thus so far been solved only in the 3-dimensional (or equal-time) formulation [7, 11].

One way [7] to obtain equal-time kinetic equations which parallels the procedure in the covariant formulation is to Wigner transform the equation of motion for the equal-time density operator $\hat{\Phi}(\boldsymbol{x}, \boldsymbol{y}, t)$. For spinor QED this procedure results in the BGR equations [7] for the equal-time Wigner functions. In Ref. [12] we suggested a different derivation which is based on taking the energy average of the covariant kinetic equations in the 4-dimensional formulation. It exploits the fact that the equal-time Wigner function is the energy average 
(i.e. zeroth order energy moment) of the covariant one. With this method we showed for spinor QED that the direct energy average of the covariant kinetic equations leads, in addition to the BGR transport equations for the spinor components of the equal-time Wigner function, also to a second group of constraint equations which couple the equal-time Wigner function to the first order energy moment of the covariant one. In the classical $(\hbar \rightarrow 0)$ limit, these additional equations provide essential constraints on the equal-time Wigner function and allow to reduce the number of independent distribution functions by a factor of two [12]. In the general quantum case, the additional equations determine the time evolution of the energy distribution function which in general can not be expressed in terms of the equal-time Wigner function. In this sense the BGR equations do not provide a complete set of equal-time kinetic equations.

As we will discuss in this paper, this incompleteness has a more general aspect. As just mentioned, the equal-time Wigner operator is related to the covariant one by 12

$$
\hat{W}(x, \boldsymbol{p})=\int d E \hat{\mathcal{W}}(x, \boldsymbol{p}, E)
$$

where we wrote $p=(E, \boldsymbol{p}), E$ independent of $\boldsymbol{p}$. As such it is only the lowest member of an infinite hierarchy of energy moments of the covariant Wigner operator:

$$
\hat{W}_{j}(x, \boldsymbol{p})=\int d E E^{j} \hat{\mathcal{W}}(x, \boldsymbol{p}, E), \quad j=0,1,2, \cdots,
$$

with $\hat{W}_{0}(x, \boldsymbol{p}) \equiv \hat{W}(x, \boldsymbol{p})$. Therefore, to set up a complete equal-time transport theory which contains the same amount of information as the covariant theory one needs dynamical equations for all the energy moments. Any covariant kinetic equation will thus correspond to an infinite hierarchy of coupled kinetic equations for its energy moments, i.e. for the equal-time Wigner operators $\hat{W}_{j}(x, \boldsymbol{p})$.

This infinite hierarchy only exists for genuine quantum problems where the energy can exhibit quantum fluctuations. In the classical limit, the covariant Wigner operator satisfies the mass-shell constraint $p^{2}=E^{2}-\boldsymbol{p}^{2}=m^{2}$, and the energy dependence of the covariant Wigner function thus degenerates to two delta-functions at $E= \pm E_{p}= \pm \sqrt{m^{2}+\boldsymbol{p}^{2}}$. The 
equal-time Wigner operator $\hat{W}(x, \boldsymbol{p})$ then splits into a positive and a negative frequency component,

$$
\hat{W}(x, \boldsymbol{p})=\hat{W}^{+}(x, \boldsymbol{p})+\hat{W}^{-}(x, \boldsymbol{p}), \quad(\hbar \rightarrow 0)
$$

and all energy moments can be expressed algebraically in terms of these as

$$
\begin{aligned}
\hat{W}_{j}(x, \boldsymbol{p}) & =\hat{W}_{j}^{+}(x, \boldsymbol{p})+\hat{W}_{j}^{-}(x, \boldsymbol{p}) \\
& =E_{p}^{j} \hat{W}^{+}(x, \boldsymbol{p})+\left(-E_{p}\right)^{j} \hat{W}^{-}(x, \boldsymbol{p}), \quad j=0,1,2, \ldots \quad(\hbar \rightarrow 0) .
\end{aligned}
$$

The solution of the equal-time kinetic equations for $\hat{W}(x, \boldsymbol{p})$ thus also determines the dynamics of all higher energy moments. Thus, in the classical limit, a simple zeroth order energy average of the covariant kinetic theory yields a complete equal-time kinetic theory.

In the general quantum case, the higher order energy moments $\hat{W}_{j}(x, \boldsymbol{p}), j \geq 1$, contain genuine additional information and can no longer be expressed algebraically through the equal-time Wigner operator $\hat{W}(x, \boldsymbol{p})$. This means that in principle in the equal-time formulation we are stuck with the problem of solving an infinite hierarchy of coupled equations. Actually, there are two such hierarchies, one resulting from the covariant transport equation ("transport hierarchy"), the other arising from the generalized mass-shell constraint ("constraint hierarchy"). In practice this raises the problem of truncating the hierarchy in a physically sensible way. Since only the low-order energy moments of the covariant Wigner function have an intuitive physical interpretation, it turns out that physics itself suggests an appropriate truncation scheme. We will show that the hierarchies of moment equations are structured in such a way that the first few low-order moments form a finite and closed subgroup of equations which can be solved as an initial value problem, and that (surprisingly) all the higher order moments can be derived from these low-order moments recursively using only the constraint hierarchy, i.e. without solving any additional equations of motion. The equations from the transport hierarchy for the higher order moments are redundant.

We first discuss on a general basis, starting from the covariant approach, the derivation and truncation of equal-time hierarchies of kinetic equations. For illustration we then 
consider in full generality the case of a transport theory for scalar fields with arbitrary scalar potentials. For this case everything can be worked out explicitly to arbitrary order of the moments. We give the subgroup of equations which fully characterize the first few low-order moments, prove the independence and redundance of the transport equations for the higher order moments outside this subgroup, and obtain from the constraint hierarchy explicit expressions for all the higher order moments in terms of the solutions of the loworder subgroup. We then apply the general formalism to scalar and spinor QED. Here the equations have a more complicated structure, and we restrict our attention to the closed subgroup of equations for the lowest order moments, discussing the redundance of the transport equations for the higher order moments and their recursive determination through the constraint hierarchy only for the first moments outside the closed subgroup of equations for the low-order moments. We will compare our results with the kinetic equations for the equal-time distribution functions obtained previously in Refs. [7, 8, 12]. Our final result will be a complete set of kinetic equations which can be implemented numerically as an initial value problem.

\section{GENERAL FORMALISM}

The 4-dimensional Wigner transform of the equation of motion for the covariant density operator leads to a complex, Lorentz covariant kinetic equation for the Wigner operator. It couples the one-body Wigner operator to two-body correlations [1], which in turn satisfy an equation which couples them to three-body terms, and so on. After taking an ensemble average this generates the so-called BBGKY hierarchy 13 for the $n$-body Wigner functions. A popular way to get a closed kinetic equation for the one-body Wigner function (i.e. the ensemble average of the one-body Wigner operator) is to truncate the BBGKY hierarchy at the one-body level, by factorizing the two-body Wigner functions in the Hartree approximation. So far most applications of quantum transport theory have employed this approximation, and in the following we will also restrict ourselves to it. For us the mean 
field approximation provides a crucial simplification, and at present it is not obvious to us how to generalize our results in order to include correlations and collision terms.

For a scalar field in mean field approximation the complex equation for the self-adjoint scalar Wigner function can be separated into two independent real equations [12]:

$$
\begin{aligned}
& \hat{G}(x, p) \mathcal{W}(x, p)=0 ; \\
& \hat{F}(x, p) \mathcal{W}(x, p)=0 .
\end{aligned}
$$

The first equation corresponds to a generalized Vlasov equation; after performing the energy average it generates a hierarchy of transport equations for the energy moments $W_{j}(x, \boldsymbol{p})$ ("transport hierarchy"). The second equation is a generalized mass-shell constraint; it generates a hierarchy of non-dynamic constraint equations ("constraint hierarchy"). Equations with the structure given in (2.1) will be the starting point for our discussion of scalar field theories in Secs. III and IVA. Factors of $p_{\mu}$ in the dynamical operators $\hat{G}(x, p)$ and $\hat{F}(x, p)$ arise from the Wigner transformation of the partial derivative $\partial_{\mu}$ in the Klein-Gordon equation. Since the latter contains at most second order time derivatives, at most two powers of $p_{0}$ occur. In fact, $\hat{G}(x, p)$ is linear in $p_{0}$ while $\hat{F}(x, p)$ is quadratic in $p_{0}$. This will be important below (see Sec. [1B).

For spinor fields the covariant Wigner function is a $4 \times 4$ matrix in spinor space which for a physical interpretation must be decomposed into its 16 spinor components $\mathcal{W}^{s}$. In this way the complex kinetic equation for the Wigner function matrix is split into 32 independent real equations for the self-adjoint spinor components [6, 12]. These equations can be further divided into two subgroups according to their anticipated structure after performing the energy average:

$$
\begin{aligned}
& \sum_{s^{\prime}=1}^{16} \hat{G}^{s s^{\prime}}(x, p) \mathcal{W}^{s^{\prime}}(x, p)=0 \\
& \sum_{s^{\prime}=1}^{16} \hat{F}^{s s^{\prime}}(x, p) \mathcal{W}^{s^{\prime}}(x, p)=0, \quad(s=1,2, \ldots, 16) .
\end{aligned}
$$

The first subgroup leads to equations containing only first order time derivatives and thus generates 16 hierarchies of transport equations for the energy moments of the spinor com- 
ponents ("transport hierarchies"). The other subgroup which involves both first and second order time derivatives leads to a set of 16 hierarchies of constraint equations ("constraint hierarchies") for the equal-time moments of the spinor components. For the lowest energy moments, the spinor components $W^{s}(x, \boldsymbol{p})$ of the equal-time Wigner function, the details of this procedure were worked out in Ref. [12], and we will use these results in Sec. [VC. Since the original Dirac equation is linear in the time derivative, the dynamical operators $\hat{G}^{s s^{\prime}}(x, p)$ and $\hat{F}^{s s^{\prime}}(x, p)$ contain at most single powers of $p_{0}$. In fact, the operators $\hat{G}^{s s^{\prime}}(x, p)$ are independent of $p_{0}$.

\section{A. Hierarchy of energy moments}

In this subsection we will concentrate for simplicity on a single covariant kinetic equation of the generic form

$$
\hat{G}(x, p) \mathcal{W}(x, p)=0
$$

where $\hat{G}(x, p)$ contains at most two powers of $p_{0}$ and of the space-time derivative operator $\partial_{\mu}$, but an arbitrary number of derivatives with respect to the momentum space coordinates (see Appendix C). We will return to the full set of equations (2.1) resp. (2.2) in the following Sections.

We begin by decomposing the energy dependence of the Wigner function into a basis of orthogonal polynomials $h_{j}(E)$ :

$$
\mathcal{W}(x, \boldsymbol{p}, E)=\sum_{j=0}^{\infty} w_{j}(x, \boldsymbol{p}) h_{j}(E) .
$$

The expansion coefficients $w_{j}(x, \boldsymbol{p})$ are defined in the equal-time phase space. Using the orthonormality relation

$$
\int d \mu(E) h_{i}(E) h_{j}(E)=\delta_{i j}
$$

where $d \mu(E)$ is the appropriate integration measure associated with the chosen set of poly-

nomials $h_{j}$, the equal-time components $w_{j}(x, \boldsymbol{p})$ can be related to energy moments of the covariant Wigner function constructed with the basis functions $h_{j}(E)$ : 


$$
w_{j}(x, \boldsymbol{p})=\int d \mu(E) h_{j}(E) \mathcal{W}(x, \boldsymbol{p}, E)
$$

If the system has finite total energy the covariant spinor components must vanish in the limit $E \rightarrow \pm \infty$. We will assume that they vanish at infinite energy faster than any power of $E$ such that for any combination of integers $i, j, m, n \geq 0$, we have

$$
\int d \mu(E) \frac{\partial}{\partial E}\left(\frac{\partial^{n}}{\partial E^{n}}\left(h_{i}(E) E^{m}\right) \frac{\partial^{j}}{\partial E^{j}} \mathcal{W}(x, \boldsymbol{p}, E)\right)=0 .
$$

With exponential accuracy we may therefore restrict the energy integration to a finite interval $-\Lambda \leq E \leq \Lambda$. Introducing the scaled energy $\omega=E / \Lambda$ we can thus use as our set of basis functions the Legendre polynomials

$$
h_{n}(\omega)=\sqrt{\frac{2 n+1}{2}} P_{n}(\omega)
$$

with the trivial measure $d \mu(\omega)=d \omega$ on the interval $[-1,1]$.

As discussed above, the dynamical operator $\hat{G}(x, p)$ in (2.3) in general contains powers of $E$ up to second order and an infinite number of energy derivatives $\partial / \partial E$. In terms of the new dimensionless energy variable we may thus write

$$
\hat{G}(x, \boldsymbol{p}, \omega)=\sum_{m=0}^{M} \sum_{n=0}^{\infty} \hat{G}_{m n}(x, \boldsymbol{p}) \omega^{m}\left(\frac{\partial}{\partial \omega}\right)^{n},
$$

with $M \leq 2$. Substituting this double expansion into equation (2.3), multiplying by $h_{i}(\omega)$ from the left and integrating over energy $\omega$ we obtain

$$
\sum_{m, n} \hat{G}_{m n}(x, \boldsymbol{p}) \int_{-1}^{1} d \omega h_{i}(\omega) \omega^{m} \frac{\partial^{n}}{\partial \omega^{n}} \mathcal{W}(x, \boldsymbol{p}, \omega)=0 .
$$

Inserting the expansion (2.4) of the covariant Wigner function $\mathcal{W}(x, \boldsymbol{p}, \omega)$, this can be written as

$$
\sum_{j=0}^{\infty} \hat{H}_{i j}(x, \boldsymbol{p}) w_{j}(x, \boldsymbol{p})=0, \quad i=0,1,2, \ldots,
$$

where

$$
\hat{H}_{i j}(x, \boldsymbol{p})=\sum_{m=0}^{M} \sum_{n=0}^{\infty} C_{i j}^{m n} \hat{G}_{m n}(x, \boldsymbol{p})
$$


with

$$
C_{i j}^{m n}=\int_{-1}^{1} d \omega h_{i}(\omega) \omega^{m} \partial_{\omega}^{n} h_{j}(\omega) .
$$

It is easy to see from $(2.13)$ that

$$
C_{i j}^{m n}=0 \quad \text { for } \quad n>j \quad \text { and } \quad i>j+m-n
$$

For $j \geq i-m+n$ the coefficients are in general nonzero. Therefore, the sum over $j$ in Eq. (2.11) extends over the range $j \geq \max [0, i-M]$. For each value of $i$, Eq. (2.11) thus contains an infinite number of terms. In this form Eqs. (2.11) are thus not practically useful. However, one can use the surface condition (2.7) to rewrite Eqs. 2.11) in such a way that each equation contains only a finite number of terms. Returning to Eq. (2.10) and integrating by parts, we can replace the integrand by

$$
\begin{aligned}
& h_{i}(\omega) \omega^{m} \partial_{\omega}^{n} \mathcal{W}(x, \boldsymbol{p}, \omega)= \\
& \sum_{l=0}^{n-1}(-)^{l} \partial_{\omega}\left(\partial_{\omega}^{l}\left(h_{i}(\omega) \omega^{m}\right) \partial_{\omega}^{n-l-1} \mathcal{W}(x, \boldsymbol{p}, \omega)\right)+(-)^{n} \partial_{\omega}^{n}\left(h_{i}(\omega) \omega^{m}\right) \mathcal{W}(x, \boldsymbol{p}, \omega) .
\end{aligned}
$$

The contribution to the integral in (2.10) from each term in the sum is fully cancelled by the surface condition (2.7), and only the last term in (2.15) survives. Inserting it into (2.10) and using again the expansion (2.4) we find instead of Eqs. (2.11-2.13) the following set of equations:

$$
\sum_{j=0}^{i+M} \hat{g}_{i j}(x, \boldsymbol{p}) w_{j}(x, \boldsymbol{p})=0, \quad i=0,1,2, \ldots
$$

with

$$
\hat{g}_{i j}(x, \boldsymbol{p})=\sum_{m=0}^{M} \sum_{n=0}^{i+m} c_{i j}^{m n} \hat{G}_{m n}(x, \boldsymbol{p})
$$

and the coefficients

$$
c_{i j}^{m n}=\frac{1}{2} \sqrt{(2 i+1)(2 j+1)} \int_{-1}^{1} d \omega P_{j}(\omega)\left(-\partial_{\omega}\right)^{n}\left(P_{i}(\omega) \omega^{m}\right) .
$$

The latter can be determined recursively from $c_{i j}^{00}=\delta_{i j}$ (which results from the orthogonality relation (2.5)) by using the recursion relations for the Legendre polynomials, see Appendix A. Since the nonvanishing coefficients $c_{i j}^{m n}$ are now restricted to the domain 


$$
n \leq i+m \text { and } j \leq i+m-n
$$

the sum over $j$ in (2.16) runs now only over the finite range $0 \leq j \leq i+M$. The first inequality in (2.19) was already used in (2.17) to limit the sum over $n$.

The $P_{i}(\omega)$ are polynomials in $\omega$ of order $i$, and thus the equal-time components $w_{j}(x, \boldsymbol{p})$ occurring in Eqs. (2.16) are linear combinations of the energy moments $W_{k}(x, \boldsymbol{p})$ of order $k \leq i$ (see Eq. (1.2)). For each value of $i$, Eq. (2.16) thus provides a relation among the first $i+M+1$ energy moments of the covariant Wigner function $\hat{W}(x, \boldsymbol{p}, \omega)$ (including the zeroth order moment $\left.W(x, \boldsymbol{p})=\sqrt{2} w_{0}(x, \boldsymbol{p})\right)$. As $i$ is allowed to run over all positive integers, Eqs. (2.16) form an infinite hierarchy of relations among the energy moments of the covariant Wigner function. Each covariant equation of the type (2.3) generates its own such hierarchy. Only the full set of these infinite hierarchies of moment equations constitutes a complete equal-time kinetic description of the system under study.

\section{B. Truncating the hierarchy}

In order to discuss possible truncation schemes we must return to the complete set of covariant kinetic equations. Let us concentrate here on the scalar case, Eqs. (2.1), and write down the two resulting hierarchies of moment equations as

$$
\begin{aligned}
\sum_{j=0}^{i+M} \hat{g}_{i j}(x, \boldsymbol{p}) w_{j}(x, \boldsymbol{p}) & =0, \\
\sum_{j=0}^{i+M+1} \hat{f}_{i j}(x, \boldsymbol{p}) w_{j}(x, \boldsymbol{p}) & =0, \quad(i=0,1,2, \ldots) .
\end{aligned}
$$

In writing down the upper limits of the sums we already used that $\hat{F}(x, p)$ in (2.1a) contains one power of $p_{0}$ more than $\hat{G}(x, p)$ in (2.1a). For the scalar field case one has $M=1$. For the spinor case one obtains from (2.2) a similar set of equations with $M=0$.

Let us now try to truncate these hierarchies for the moments $w_{j}$ at some order $j_{\max }$. The equations from the "transport hierarchy" (2.20a) with hierarchy index $i \leq I_{t}$ involve all moments $w_{j}$ with $0 \leq j \leq I_{t}+M$, i.e. the first $I_{t}+M+1$ moments (including the lowest 
moment with index 0). Similarly, the equations from the "constraint hierarchy" (2.20b) with hierarchy index $i \leq I_{c}$ involve the first $I_{c}+M+2$ moments $0 \leq j \leq I_{c}+M+1$. For a closed set of equations both hierarchies must be truncated at the same order $j_{\max }$, i.e. we must have

$$
I_{t}+M=I_{c}+M+1=j_{\max }
$$

Truncating in this way we are left with $I_{t}+1$ equations from the transport hierarchy and $I_{c}+1$ equations from the constraint hierarchy. In order solve them the number of equations must at least equal the number of moments. However, if there are more equations than moments, the system may be overdetermined, and therefore we would like to require equality of the number of equations and moments:

$$
I_{t}+1+I_{c}+1=j_{\max }+1
$$

The two conditions (2.21) and (2.22) have a unique solution:

$$
I_{t}=I_{c}+1=M, \quad j_{\max }=2 M
$$

which yields $M+1$ transport and $M$ constraint equations for the first $2 M+1$ energy moments. Smaller values of $j_{\max }$ don't yield enough equations, and larger values lead to an (at least superficially) overdetermined system of equations. For spinor fields $(M=0)$ the truncated set involves only one transport and no constraint equation; for each spinor component it gives a single kinetic equation (the BGR equation [7]) for its lowest energy moment, the equal-time Wigner function $W^{s}(x, \boldsymbol{p})$. For scalar fields $(M=1)$ the truncated set contains two transport equations and one constraint for the three lowest order moments $w_{0}, w_{1}, w_{2}$.

If we go beyond this minimal closed subset of moments and equations, we get two equations for every additional moment, one from the transport hierarchy and one from the constraint hierarchy. As we will show explicitly in the next Section, in the constraint hierarchy (2.20b) the highest moment always comes with a constant coefficient. As we increase 
the hierarchy index $i$ in Eqs. (2.20), at each step the newly occurring moment can thus be explicitly expressed in terms of the already known lower order moments using the corresponding constraint equation from (2.20b). As we will discuss, these higher order constraint equations contain important physics. But in addition, at each step there is also a dynamical equation of motion for the new moment from the transport hierarchy (2.20a). How can the two equations be consistent? The answer is that this transport equation is not an independent new equation, but (with some algebraic effort) can be expressed as a combination of the lower order equations which have already been used. Our proof of this fact uses explicitly

the structure of the dynamical operators $\hat{G}_{m n}$ and $\hat{F}_{m n}$. It involves cumbersome algebra, and only for scalar fields with only scalar potential or mean field interactions we have been able to find a general proof. For scalar and spinor QED the proof is still incomplete, and we will only demonstrate the first step for the $2 M+2^{\text {nd }}$ moment. A completion of the proof presumably requires a so far missing deeper insight into the general dynamic structure of the moment equations and their relation to the underlying covariant theory.

\section{SCALAR FIELD THEORY}

In this Section we will give an explicit and complete discussion of the moment hierarchy for the simplest case of a scalar field theory in Hartree approximation. We exemplify the truncation of the hierarchy and the recursive computation of the higher order moments beyond minimal truncation. The discussion in the following Section for the practically more relevant case of QED will be technically more involved and, unfortunately, also less complete.

\section{A. Covariant kinetic equations}

Consider the Klein-Gordon equation with a scalar potential $U(x)$ :

$$
\left(\partial_{x}^{2}+m_{0}^{2}+U(x)\right) \hat{\phi}(x)=0
$$


The covariant Wigner function is the four-dimensional Wigner transform of the covariant density matrix $\Phi(x, y)=\langle\hat{\Phi}(x, y)\rangle$ :

$$
\mathcal{W}(x, p)=\int d^{4} y e^{i p \cdot y} \Phi(x, y)=\int d^{4} y e^{i p \cdot y}\left\langle\hat{\phi}\left(x+\frac{y}{2}\right) \hat{\phi}^{+}\left(x-\frac{y}{2}\right)\right\rangle .
$$

To derive the kinetic equations for the scalar Wigner function, we calculate the second-order derivatives $\left(\frac{1}{2} \partial_{\mu}^{x}+\partial_{\mu}^{y}\right)^{2}$ and $\left(\frac{1}{2} \partial_{\mu}^{x}-\partial_{\mu}^{y}\right)^{2}$ of the covariant density operator, and then employ the Klein-Gordon equation (3.1) and its adjoint. After taking the ensemble average and performing the Wigner transform we obtain two complex kinetic equations:

$$
\begin{aligned}
& \left(\frac{1}{4} \partial_{x}^{2}-p^{2}+m_{0}^{2}+U\left(x-\frac{i}{2} \partial_{p}\right)-i p \cdot \partial_{x}\right) \mathcal{W}(x, p)=0 \\
& \left(\frac{1}{4} \partial_{x}^{2}-p^{2}+m_{0}^{2}+U\left(x+\frac{i}{2} \partial_{p}\right)+i p \cdot \partial_{x}\right) \mathcal{W}(x, p)=0
\end{aligned}
$$

Since the scalar Wigner function is real, adding and subtracting these two complex equations yields two real equations of the type (2.1). After reinstating $\hbar$ the corresponding operators $\hat{G}$ and $\hat{F}$ are given by

$$
\begin{aligned}
& \hat{G}(x, p)=\hbar p \cdot \partial_{x}+\operatorname{Im} \hat{M}^{2}(x, p), \\
& \hat{F}(x, p)=-p^{2}+\frac{\hbar^{2}}{4} \partial_{x}^{2}+\operatorname{Re} \hat{M}^{2}(x, p),
\end{aligned}
$$

where the mass operator $\hat{M}^{2}$ is defined as

$$
\begin{aligned}
\hat{M}^{2}(x, p) & =m_{0}^{2}+\hat{\Sigma}_{e}(x, p)+i \hat{\Sigma}_{o}(x, p), \\
\hat{\Sigma}_{e}(x, p) & =\cos \left(\frac{\hbar \triangle}{2}\right) U(x), \\
\hat{\Sigma}_{o}(x, p) & =\sin \left(\frac{\hbar \triangle}{2}\right) U(x) .
\end{aligned}
$$

Here the triangle operator $\triangle$ is defined as $\triangle=\partial_{x} \cdot \partial_{p}$ where the coordinate derivative $\partial_{x}$ acts only on the scalar potential $U(x)$ while $\partial_{p}$ acts only on the Wigner function.

\section{B. Semiclassical expansion}

In the general quantum situation the particles have no definite mass due to quantum fluctuations around their classical mass shell and collision effects in the medium. In the 
situation here with only an external potential this is illustrated by the mass operator $\hat{M}^{2}$. Only in the classical limit $\hbar \rightarrow 0$ it reduces to the quasiparticle mass

$$
\begin{aligned}
& \operatorname{Re} \hat{M}_{0}^{2}(x, p)=m^{2}(x)=m_{0}^{2}+U(x), \\
& \operatorname{Im} \hat{M}_{0}^{2}(x, p)=0
\end{aligned}
$$

In this case the constraint equation reduces to the on-shell condition

$$
\left(p^{2}-m^{2}(x)\right) \mathcal{W}_{0}(x, p)=0
$$

for the classical covariant Wigner function $\mathcal{W}_{0}$. The classical transport equation arises from the general transport equation at first order in $\hbar$. The first order contribution to the mass operator is

$$
\begin{aligned}
& \operatorname{Re} \hat{M}_{1}^{2}(x, p)=0, \\
& \operatorname{Im} \hat{M}_{1}^{2}(x, p)=\hbar m(x)\left(\partial_{x} m(x)\right) \cdot \partial_{p},
\end{aligned}
$$

and we obtain the covariant Vlasov equation

$$
\left(p \cdot \partial_{x}+m(x)\left(\partial_{x} m(x)\right) \cdot \partial_{p}\right) \mathcal{W}_{0}(x, p)=0,
$$

with a Vlasov force term induced by $x$-dependent effective mass term. For scalar fields there is no first order quantum correction to the operator $F$ in (3.4), and from the zeroth order term we obtain the mass-shell condition for the first order Wigner function:

$$
\left(p^{2}-m^{2}(x)\right) \mathcal{W}_{1}(x, p)=0 .
$$

This discussion holds universally for arbitrary potentials. If, for instance, $U(x)$ is generated by the scalar field $\hat{\phi}(x)$ itself in Hartree approximation,

$$
U(x)=-C+\lambda\left\langle\hat{\phi}(x) \hat{\phi}^{+}(x)\right\rangle=-C+\lambda \int \frac{d^{4} p}{(2 \pi)^{4}} \mathcal{W}(x, p),
$$

with a mass parameter $C$ and a coupling strength $\lambda$, this model provides a useful tool for a dynamical description of spontaneous symmetry breaking [8]. 


\section{The 3-dimensional dynamical operators}

We now perform the energy average of the covariant transport and constraint equations (2.1) and construct the hierarchy $(2.20)$ of moment equations. The first step is the double expansion of the type (2.9) for the covariant dynamical operators $\hat{G}(x, p)$ and $\hat{F}(x, p)$ :

$$
\begin{aligned}
& \hat{G}_{m n}(\boldsymbol{x}, \boldsymbol{p}, t)= \begin{cases}\hbar \Lambda \partial_{t} & \text { for } m=1, n=0 \\
\hbar \boldsymbol{p} \cdot \boldsymbol{\nabla}_{x}-\hat{\sigma}_{o} & \text { for } m=n=0 \\
-\frac{1}{n !}\left(\frac{i \hbar}{2 \Lambda}\right)^{n}\left(\partial_{t}^{n} \hat{\sigma}_{o}\right) & \text { for } m=0, n \neq 0 \text { even } \\
-\frac{i}{n !}\left(\frac{i \hbar}{2 \Lambda}\right)^{n}\left(\partial_{t}^{n} \hat{\sigma}_{e}\right) & \text { for } m=0, n \text { odd } \\
0 & \text { else }\end{cases} \\
& \hat{F}_{m n}(\boldsymbol{x}, \boldsymbol{p}, t)= \begin{cases}-\Lambda^{2} & \text { for } m=2, n=0 \\
\frac{\hbar^{2}}{4} \partial_{x}^{2}+\boldsymbol{p}^{2}+m_{0}^{2}+\hat{\sigma}_{e} & \text { for } m=n=0 \\
\frac{1}{n !}\left(\frac{i \hbar}{2 \Lambda}\right)^{n}\left(\partial_{t}^{n} \hat{\sigma}_{e}\right) & \text { for } m=0, n \neq 0 \text { even } \\
-\frac{i}{n !}\left(\frac{i \hbar}{2 \Lambda}\right)^{n}\left(\partial_{t}^{n} \hat{\sigma}_{o}\right) & \text { for } m=0, n \text { odd } \\
0 & \text { else. }\end{cases}
\end{aligned}
$$

Here

$$
\begin{aligned}
& \hat{\sigma}_{e}(\boldsymbol{x}, \boldsymbol{p}, t)=\cos \left(\frac{\hbar}{2} \boldsymbol{\nabla}_{x} \cdot \nabla_{p}\right) U(\boldsymbol{x}, t), \\
& \hat{\sigma}_{o}(\boldsymbol{x}, \boldsymbol{p}, t)=\sin \left(\frac{\hbar}{2} \boldsymbol{\nabla}_{x} \cdot \nabla_{p}\right) U(\boldsymbol{x}, t)
\end{aligned}
$$

are the three-dimensional analogues of the covariant operators $\hat{\Sigma}_{e}$ and $\hat{\Sigma}_{o}$ in Eq. (3.5). Again, the spatial gradients act only on $U(\boldsymbol{x}, t)$, while the momentum gradients act on the equal-time Wigner functions (i.e. on the energy moments $w_{j}(x, \boldsymbol{p})$ ).

The three-dimensional dynamical operators $\hat{G}_{m n}(x, \boldsymbol{p})$ and $\hat{F}_{m n}(x, \boldsymbol{p})$ must now be combined with the coefficients $c_{i j}^{m n}$ to obtain the dynamical operators $\hat{g}_{i j}(x, \boldsymbol{p})$ and $\hat{f}_{i j}(x, \boldsymbol{p})$ which are needed in the transport and constraint hierarchies. This is done in Appendix B. 


\section{Minimal truncation}

The resulting transport hierarchy is truncated at $I_{t}=M=1$, the constraint hierarchy at $I_{c}=M-1=0$. This yields the following equations for $w_{0}(x, \boldsymbol{p}), w_{1}(x, \boldsymbol{p})$, and $w_{2}(x, \boldsymbol{p})$ :

$$
\begin{aligned}
& \hat{g}_{00} w_{0}+\hat{g}_{01} w_{1}=0, \\
& \hat{g}_{10} w_{0}+\hat{g}_{11} w_{1}+\hat{g}_{12} w_{2}=0, \\
& \hat{f}_{00} w_{0}+\hat{f}_{01} w_{1}+\hat{f}_{02} w_{2}=0 .
\end{aligned}
$$

The dynamical operators $\hat{g}_{i j}$ and $\hat{f}_{i j}$ are given in Appendix B and Eqs. (3.12). Reexpressing $w_{j}$ in terms of the energy moments $W_{j}$ from Eq. (1.2),

$$
\begin{aligned}
& w_{0}(x, \boldsymbol{p})=\frac{1}{\Lambda \sqrt{2}} W(\boldsymbol{x}, \boldsymbol{p}, t), \\
& w_{1}(x, \boldsymbol{p})=\frac{1}{\Lambda^{2}} \sqrt{\frac{3}{2}} W_{1}(\boldsymbol{x}, \boldsymbol{p}, t), \\
& w_{2}(x, \boldsymbol{p})=\frac{1}{2 \Lambda} \sqrt{\frac{5}{2}}\left(\frac{3}{\Lambda^{2}} W_{2}(\boldsymbol{x}, \boldsymbol{p}, t)-W(\boldsymbol{x}, \boldsymbol{p}, t)\right),
\end{aligned}
$$

Eqs. (3.14) can be rewritten as

$$
\begin{aligned}
\partial_{t} W_{1}(\boldsymbol{x}, \boldsymbol{p}, t) & =-\left(\boldsymbol{p} \cdot \nabla_{x}-\frac{1}{\hbar} \hat{\sigma}_{o}(\boldsymbol{x}, \boldsymbol{p}, t)\right) W(\boldsymbol{x}, \boldsymbol{p}, t), \\
\partial_{t} W_{2}(\boldsymbol{x}, \boldsymbol{p}, t) & =-\left(\boldsymbol{p} \cdot \nabla_{x}-\frac{1}{\hbar} \hat{\sigma}_{o}(\boldsymbol{x}, \boldsymbol{p}, t)\right) W_{1}(\boldsymbol{x}, \boldsymbol{p}, t)+\frac{1}{2}\left(\partial_{t} \hat{\sigma}_{e}(\boldsymbol{x}, \boldsymbol{p}, t)\right) W(\boldsymbol{x}, \boldsymbol{p}, t), \\
W_{2}(\boldsymbol{x}, \boldsymbol{p}, t) & =\left(\frac{\hbar^{2}}{4}\left(\partial_{t}^{2}-\nabla_{x}^{2}\right)+\boldsymbol{p}^{2}+m_{0}^{2}+\hat{\sigma}_{e}(\boldsymbol{x}, \boldsymbol{p}, t)\right) W(\boldsymbol{x}, \boldsymbol{p}, t) .
\end{aligned}
$$

Please note that all powers of the cutoff $\Lambda$ cancel in the final expressions as they should.

The two transport equations (3.16a,b) do not decouple, not even in the classical limit $\hbar \rightarrow 0$. To achieve decoupling one must return to the covariant equations in Sec. IIIA and study their semiclassical limit as given in Sec. [IIB before performing the energy average. Then the mass-shell condition (3.7) can be used to rewrite all higher order energy moments

in terms of the zeroth order moment as explained in the Introduction, Eq. (1.4). With this information the constraint (3.160), in the limit $\hbar \rightarrow 0$, becomes trivial, 


$$
W_{2}(\boldsymbol{x}, \boldsymbol{p}, t)=E_{p}^{2}(\boldsymbol{x}, t) W(\boldsymbol{x}, \boldsymbol{p}, t), \quad E_{p}^{2}(\boldsymbol{x}, t)=\boldsymbol{p}^{2}+m^{2}(\boldsymbol{x}, t)
$$

while the two transport equations (3.16a) and (3.16b) become identical and can be written in the form of a Vlasov equation for the charge density (see Sec. IV):

$$
\partial_{t} W_{1}(\boldsymbol{x}, \boldsymbol{p}, t)+\left(\frac{\boldsymbol{p}}{E_{p}} \cdot \nabla_{x}-\nabla_{x} E_{p} \cdot \nabla_{p}\right) W_{1}(\boldsymbol{x}, \boldsymbol{p}, t)=0 .
$$

The reason why the information contained in Eq. (1.4) cannot be easily recovered directly from the 3-dimensional transport and constraint equations is that in their derivation, through Eq. (2.15), we made heavy use of partial integration with respect to the energy. In the

classical limit this has the unfortunate effect of spreading the information contained in the on-shell condition over the whole infinite hierarchy of 3-dimensional constraint equations.

Although we have always talked about Eq. (3.16c) as a "constraint equation" it is clear that, as far as solving the minimal subset (3.16) of equal-time kinetic equations is concerned, this terminology is only adequate in the classical limit $\hbar \rightarrow 0$. In general quantum situations it is a second order partial differential equation for the lowest order moment $W(\boldsymbol{x}, \boldsymbol{p}, t)$ which must be solved as an initial value problem together with the first order partial differential equations (3.16a) and (3.16b).

\section{E. Higher order moment equations}

The next higher moment $w_{3}$ is determined by the third equation in the transport hierarchy and the second equation in the constraint hierarchy,

$$
\begin{aligned}
& \hat{g}_{20} w_{0}+\hat{g}_{21} w_{1}+\hat{g}_{22} w_{2}+\hat{g}_{23} w_{3}=0 \\
& \hat{f}_{10} w_{0}+\hat{f}_{11} w_{1}+\hat{f}_{12} w_{2}+\hat{f}_{13} w_{3}=0
\end{aligned}
$$

With the dynamical operators from Appendix B and Eqs. (3.12) and

$$
w_{3}(x, \boldsymbol{p})=\frac{1}{2 \Lambda} \sqrt{\frac{7}{2}}\left(\frac{5}{\Lambda^{3}} W_{3}(\boldsymbol{x}, \boldsymbol{p}, t)-\frac{3}{\Lambda} W_{1}(\boldsymbol{x}, \boldsymbol{p}, t)\right)
$$

we obtain (using Eq. (3.16a)) 


$$
\begin{aligned}
\partial_{t} W_{3}(\boldsymbol{x}, \boldsymbol{p}, t)= & -\left(\boldsymbol{p} \cdot \nabla_{x}-\frac{1}{\hbar} \hat{\sigma}_{o}(\boldsymbol{x}, \boldsymbol{p}, t)\right) W_{2}(\boldsymbol{x}, \boldsymbol{p}, t)+\left(\partial_{t} \hat{\sigma}_{e}(\boldsymbol{x}, \boldsymbol{p}, t)\right) W_{1}(\boldsymbol{x}, \boldsymbol{p}, t) \\
& -\frac{\hbar}{4}\left(\partial_{t}^{2} \hat{\sigma}_{o}(\boldsymbol{x}, \boldsymbol{p}, t)\right) W(\boldsymbol{x}, \boldsymbol{p}, t), \\
W_{3}(\boldsymbol{x}, \boldsymbol{p}, t)= & \left(\frac{\hbar^{2}}{4}\left(\partial_{t}^{2}-\nabla_{x}^{2}\right)+\boldsymbol{p}^{2}+m_{0}^{2}+\hat{\sigma}_{e}(\boldsymbol{x}, \boldsymbol{p}, t)\right) W_{1}(\boldsymbol{x}, \boldsymbol{p}, t) \\
& -\frac{\hbar}{2}\left(\partial_{t} \hat{\sigma}_{o}(\boldsymbol{x}, \boldsymbol{p}, t)\right) W(\boldsymbol{x}, \boldsymbol{p}, t) .
\end{aligned}
$$

By substituting (3.19b) into (3.19a) and taking into account the following commutators:

$$
\begin{aligned}
& {\left[\hat{G}_{10}, \hat{F}_{00}\right]=-2 \hat{G}_{01} \hat{F}_{20},} \\
& {\left[\hat{G}_{00}, \hat{F}_{00}\right]=\hat{F}_{01} \hat{G}_{10}-2 \hat{G}_{02} \hat{F}_{20},} \\
& {\left[\frac{\hbar^{2}}{4} \partial^{2}, \hat{\sigma}_{e / o}\right]=\frac{\hbar^{2}}{2} \partial \hat{\sigma}_{e / o} \cdot \partial+\frac{\hbar^{2}}{4} \partial^{2} \hat{\sigma}_{e / o},} \\
& {\left[\boldsymbol{p} \cdot \nabla_{x}, \hat{\sigma}_{e / o}\right]=\boldsymbol{p} \cdot \nabla_{x} \hat{\sigma}_{e / o}+\frac{\hbar}{2} \nabla_{x} \hat{\sigma}_{o / e} \cdot \nabla_{x},} \\
& {\left[\boldsymbol{p}^{2}, \hat{\sigma}_{e / o}\right]=-\hbar \boldsymbol{p} \cdot \boldsymbol{\nabla}_{x} \hat{\sigma}_{o / e}+\frac{\hbar^{2}}{4} \boldsymbol{\nabla}_{x}^{2} \hat{\sigma}_{e / o},}
\end{aligned}
$$

the third transport equation (3.19a) can be rewritten in terms of the first transport equation (3.14a) as

$$
\hat{f}_{00}\left(\hat{g}_{00} w_{0}+\hat{g}_{01} w_{1}\right)=0
$$

This implies that the transport equation (3.19a) for $w_{3}$ is redundant. The third-order moment $W_{3}$ is completely determined in terms of the solutions of the minimal subgroup (3.16) by the constraint equation (3.21b). It arises from Eq. (3.19b) by noting that $\hat{f}_{13}$ is a constant, $\hat{f}_{13}=C_{3}=-\frac{2 \sqrt{3}}{5 \sqrt{7}} \Lambda^{2}$ (see Appendix B), and solving for $w_{3}$ :

$$
w_{3}=-\frac{1}{C_{3}}\left(\hat{f}_{10} w_{0}+\hat{f}_{11} w_{1}+\hat{f}_{12} w_{2}\right) \text {. }
$$

Note that, in contrast to (3.16d), Eq. (3.21b) does not require solving a partial differential equation because everything on the r.h.s. is known from the solution of Eqs. (3.16).

The above procedure can be extended to all higher order moment equations, by repeatedly using the commutators listed in (3.22). In general one finds that a transport equation

$$
\sum_{j=0}^{i+1} \hat{g}_{i j} w_{j}=0
$$


with $i \geq 2$ can be re-expressed in terms of the first $(i-1)$ transport equations as

$$
\sum_{j=0}^{i-2} \hat{f}_{i-2, j}\left(\sum_{k=0}^{j+1} \hat{g}_{j k} w_{k}\right)=0
$$

Thus, except for the first two, all transport equations are redundant. The higher order moments $w_{i}$ with $i>2$ can be computed from their constraint equations

$$
w_{i}=-\frac{1}{C_{i}} \sum_{j=0}^{i-1} \hat{f}_{i-2, j} w_{j}
$$

with the constant $C_{i}$ given by

$$
C_{i} \equiv \hat{f}_{i-2, i}=-\frac{i(i-1)}{(2 i-1) \sqrt{(2 i-3)(2 i+1)}}
$$

\section{APPLICATION TO QED}

\section{A. Scalar QED}

In this Section we discuss the application of the general formalism developed in Sec. 凹I to QED. Since some of the equations will be rather lengthy we will economize on the notation by dropping all factors of $\hbar$. The latter are correctly given in Refs. [12,17] to which we refer in case of need.

We begin with the case of scalar QED with external electromagnetic fields. In Ref. [12 we discussed the semiclassical transport equations for this theory by energy averaging the semiclassical limit of the covariant transport equations. In this subsection we will derive the general equal-time quantum transport equations by performing the energy average without any approximations. In the following subsection the result will be compared with the corresponding equations derived by directly Wigner-transforming the equations of motion for the equal-time density matrix [8].

In scalar QED the scalar field obeys the Klein-Gordon equation

$$
\left(\left(\partial_{\mu}+i e A_{\mu}(x)\right)\left(\partial^{\mu}+i e A^{\mu}(x)\right)+m^{2}\right) \phi(x)=0
$$


Following an analogous procedure as in Sec. [IIA (see [12 for details) one derives two covariant kinetic equations of type (2.1) for the covariant Wigner function

$$
\mathcal{W}(x, p)=\int d^{4} y e^{i p \cdot y}\left\langle\hat{\phi}\left(x+\frac{y}{2}\right) \exp \left[i e \int_{-1 / 2}^{1 / 2} d s A(x+s y) \cdot y\right] \hat{\phi}^{+}\left(x-\frac{y}{2}\right)\right\rangle .
$$

The corresponding covariant dynamical operators $\hat{G}$ and $\hat{F}$ are given by

$$
\begin{aligned}
\hat{G}(x, p) & =\hat{\Pi}^{\mu}(x, p) \hat{D}_{\mu}(x, p) \\
\hat{F}(x, p) & =\frac{1}{4} \hat{D}^{\mu}(x, p) \hat{D}_{\mu}(x, p)-\hat{\Pi}^{\mu}(x, p) \hat{\Pi}_{\mu}(x, p)+m^{2} \\
\hat{\Pi}_{\mu}(x, p) & =p_{\mu}-i e \int_{-\frac{1}{2}}^{\frac{1}{2}} d s s F_{\mu \nu}\left(x-i s \partial_{p}\right) \partial_{p}^{\nu} \\
\hat{D}_{\mu}(x, p) & =\partial_{\mu}-e \int_{-\frac{1}{2}}^{\frac{1}{2}} d s F_{\mu \nu}\left(x-i s \partial_{p}\right) \partial_{p}^{\nu}
\end{aligned}
$$

$F^{\mu \nu}=\partial^{\mu} A^{\nu}-\partial^{\nu} A^{\mu}$ is the electromagnetic field tensor.

The structure of the equal-time transport theory for scalar QED is very similar to that for a scalar potential $U(x)$ which we considered in the previous section. The difference resides solely in the expressions for the dynamical operators $\hat{G}_{m n}$ and $\hat{F}_{m n}$. In Appendix 9 we provide the double expansions (2.9) for the basic operators $\hat{\Pi}^{\mu}$ and $\hat{D}^{\mu}$ as well as for $\hat{G}$ and $\hat{F}$.

In terms of the covariant Wigner function the charge current $j^{\mu}$ and the energymomentum tensor $T^{\mu \nu}$ are expressed as

$$
\begin{aligned}
& j^{\mu}(x, p)=e p^{\mu} \mathcal{W}(x, p), \\
& T^{\mu \nu}(x, p)=p^{\mu} p^{\nu} \mathcal{W}(x, p) .
\end{aligned}
$$

After performing the energy average these equations translate into relations between the first three moments $w_{0}, w_{1}, w_{2}$ and the equal-time phase-space distributions for the scalar density $W(\boldsymbol{x}, \boldsymbol{p}, t)$, the charge density $\rho(\boldsymbol{x}, \boldsymbol{p}, t)$, and the energy density $\epsilon(\boldsymbol{x}, \boldsymbol{p}, t)$ :

$$
\begin{aligned}
w_{0}(\boldsymbol{x}, \boldsymbol{p}, t) & =\frac{1}{\Lambda \sqrt{2}} W(\boldsymbol{x}, \boldsymbol{p}, t) \\
w_{1}(\boldsymbol{x}, \boldsymbol{p}, t) & =\sqrt{\frac{3}{2}} \frac{1}{e \Lambda^{2}} \rho(\boldsymbol{x}, \boldsymbol{p}, t), \\
w_{2}(\boldsymbol{x}, \boldsymbol{p}, t) & =\frac{1}{2 \Lambda} \sqrt{\frac{5}{2}}\left(\frac{3}{\Lambda^{2}} \epsilon(\boldsymbol{x}, \boldsymbol{p}, t)-W(\boldsymbol{x}, \boldsymbol{p}, t)\right) .
\end{aligned}
$$


The charge current density $\boldsymbol{j}(\boldsymbol{x}, \boldsymbol{p}, t)$ and the momentum density $\boldsymbol{P}(\boldsymbol{x}, \boldsymbol{p}, t)$ can be expressed in terms of $W$ and $\rho$ as

$$
\boldsymbol{j}(\boldsymbol{x}, \boldsymbol{p}, t)=e \boldsymbol{p} W(\boldsymbol{x}, \boldsymbol{p}, t), \quad \boldsymbol{P}(\boldsymbol{x}, \boldsymbol{p}, t)=\frac{\boldsymbol{p}}{e} \rho(\boldsymbol{x}, \boldsymbol{p}, t) .
$$

The subgroup (3.14) determining the moments $w_{0}, w_{1}$ and $w_{2}$ can thus be equivalently rewritten as transport and constraint equations for $W, \rho$ and $\epsilon$ :

$$
\begin{aligned}
& \frac{1}{e} \hat{d}_{t} \rho+\left(\hat{d}_{t} \hat{\pi}_{0}+\hat{\boldsymbol{\pi}} \cdot \hat{\boldsymbol{d}}\right) W=0, \\
& \hat{d}_{t} \epsilon+\frac{1}{e}\left(\hat{d}_{t} \hat{\pi}_{0}+\hat{\boldsymbol{\pi}} \cdot \hat{\boldsymbol{d}}+\hat{D}\right) \rho+\left(\hat{\pi}_{0} \hat{D}+\hat{\boldsymbol{\pi}} \cdot \hat{\boldsymbol{I}}-\hat{d}_{t} \hat{A}+\hat{\boldsymbol{G}} \cdot \hat{\boldsymbol{d}}\right) W=0, \\
& \epsilon=\left(\frac{1}{4}\left(\hat{d}_{t}^{2}-\hat{\boldsymbol{d}}^{2}\right)-\left(\hat{\pi}_{0}^{2}-\hat{\boldsymbol{\pi}}^{2}\right)+m^{2}+\hat{A}\right) W-\frac{2 \hat{\pi}_{0}}{e} \rho,
\end{aligned}
$$

The expressions of $\hat{g}_{i j}$ and $\hat{f}_{i j}$ were obtained via the relations given in Appendix B from the equal-time operators (C2) in Appendix Q. We have also used the commutators

$$
\begin{aligned}
{\left[\hat{d}_{t}, \hat{\pi}_{0}\right] } & =\hat{D}, \\
{\left[\hat{d}_{t},\right.} & \hat{A}]=2 \hat{E} .
\end{aligned}
$$

Due to the line integrals over $s$ in the operators $\hat{\Pi}_{\mu}$ and $\hat{D}_{\mu}$ which guarantee the gauge invariance of the formalism [1], the equal-time operators $\hat{G}_{m n}$ and $\hat{F}_{m n}$ for QED are much more complicated than Eqs. (3.12) for the case of a scalar potential. This is the origin of the more complicated structure of the minimal subgroup (4.7) of 3-dimensional kinetic equations. Please note the sequence of the operators in Eqs. (4.7): in particular the generalized time derivatives $\hat{d}_{t}$ act on everything following them. Eqs. (4.7) are thus much more intricately coupled than the corresponding equations (3.16) for the pure scalar case.

It is instructive to integrate Eqs. (4.7) over $\boldsymbol{p}$ to obtain equations of motion for the corresponding space-time densities:

$$
\begin{aligned}
& \partial_{t} \rho(\boldsymbol{x}, t)+\boldsymbol{\nabla} \cdot \boldsymbol{j}(\boldsymbol{x}, t)=0, \\
& \partial_{t} \epsilon(\boldsymbol{x}, t)+\boldsymbol{\nabla} \cdot \boldsymbol{P}(\boldsymbol{x}, t)-\boldsymbol{E}(\boldsymbol{x}, t) \cdot \boldsymbol{j}(\boldsymbol{x}, t)=0, \\
& \epsilon(\boldsymbol{x}, t)=\int \frac{d^{3} p}{(2 \pi)^{3}}\left(\frac{\hbar^{2}}{4}\left(\partial_{t}^{2}-\nabla_{x}^{2}\right)+\boldsymbol{p}^{2}+m^{2}\right) W(\boldsymbol{x}, \boldsymbol{p}, t) .
\end{aligned}
$$


The first two equations express the conservation of electric charge and of energy-momentum while the last equation gives the energy density in terms of the scalar equal-time phase-space density $W(\boldsymbol{x}, \boldsymbol{p}, t)$ including quantum corrections. In Eq. (4.9b) $\boldsymbol{P}(\boldsymbol{x}, t)=$ $\int\left(d^{3} p /(2 \pi)^{3}\right) \boldsymbol{P}(\boldsymbol{x}, \boldsymbol{p}, t)$ is the momentum density in coordinate space, and the last term describes the conversion of field energy into mechanical energy by the work done by the electric field on moving charges.

Let us now consider the next two equations in the hierarchy, the transport and constraint equations (3.19) for the third-order moment $w_{3}$. Again the constraint equation can be directly solved for $w_{3}$ in terms of the solutions $w_{0}, w_{1}, w_{2}$ of Eqs. (4.5), (4.7) (see Eq. (3.24)). Our task is to show that the transport equation for $w_{3}$ is redundant. To this end we substitute the constraint (3.19b) into the transport equation (3.19a) and use the scalar QED analogue of the commutators (3.22), namely (4.8) and

$$
\begin{aligned}
& {\left[\hat{d}_{t}, \hat{B}\right]=3 \hat{F}} \\
& {\left[\hat{d}_{t}, \hat{\boldsymbol{\pi}}\right]=-\boldsymbol{I}+\boldsymbol{\nabla}_{x} \hat{\pi}_{0},} \\
& {[\hat{\boldsymbol{I}}, \hat{\boldsymbol{\pi}}]=-\nabla_{x} \cdot \hat{\boldsymbol{G}}} \\
& {\left[\hat{\boldsymbol{d}}, \hat{\pi}_{0}\right]=\nabla_{x} \hat{\pi}_{0}} \\
& {\left[\hat{d}_{t}, \hat{\boldsymbol{d}}\right]=-e \boldsymbol{\nabla}_{x} \cdot \boldsymbol{\nabla}_{p} \int_{-\frac{1}{2}}^{\frac{1}{2}} d s \boldsymbol{E}\left(\boldsymbol{x}+i s \boldsymbol{\nabla}_{p}, t\right),} \\
& {\left[\hat{\boldsymbol{\pi}}, \hat{\pi}_{0}\right]=-i e \int_{-\frac{1}{2}}^{\frac{1}{2}} d s s \boldsymbol{E}\left(\boldsymbol{x}+i s \boldsymbol{\nabla}_{p}, t\right)+e \boldsymbol{\nabla}_{x} \int_{-\frac{1}{2}}^{\frac{1}{2}} d s s^{2} \boldsymbol{E}\left(\boldsymbol{x}+i s \boldsymbol{\nabla}_{p}, t\right) \cdot \boldsymbol{\nabla}_{p},}
\end{aligned}
$$

as well as the identity

$$
\int_{-\frac{1}{2}}^{\frac{1}{2}} d s\left(2 i s+\frac{1}{4} \boldsymbol{\nabla}_{x} \cdot \boldsymbol{\nabla}_{p}-s^{2} \boldsymbol{\nabla}_{x} \cdot \boldsymbol{\nabla}_{p}\right) \boldsymbol{E}\left(\boldsymbol{x}+i s \boldsymbol{\nabla}_{p}, t\right)=0
$$

to rewrite $(3.19 \mathrm{a})$ in the form

$$
\hat{f}_{00}\left(\hat{g}_{00} w_{0}+\hat{g}_{01} w_{1}\right)+\hat{f}_{01}\left(\hat{g}_{10} w_{0}+\hat{g}_{11} w_{1}+\hat{g}_{12} w_{2}\right)=0
$$

Note that in the derivation of the commutators (4.10) we used Maxwell's equation

$$
\partial_{\mu} \tilde{F}^{\mu \nu}=0
$$


for the dual field strength tensor $\tilde{F}_{\mu \nu}=\frac{1}{2} \epsilon_{\mu \nu \sigma \rho} F^{\sigma \rho}$. The identity 4.11) (which has no analogue in a theory without gauge invariance) is proven by expanding the electric field $\boldsymbol{E}\left(\boldsymbol{x}+i s \boldsymbol{\nabla}_{p}, t\right)=e^{i s \boldsymbol{\nabla}_{x} \cdot \boldsymbol{\nabla}_{p}} \boldsymbol{E}(\boldsymbol{x}, t)$ and integrating term by term. Eq. (4.12) expresses the transport equation for $w_{3}$ in terms of the transport equations for $w_{1}$ and $w_{2}$. This proves that it is redundant.

¿From the comparison of (4.12) with $(3.23)$ we observe that in the case of scalar QED the third transport equation is expressed in terms of the first and second transport equations, while only the first one occurs in the case of scalar potentials. This difference results from the line integrals which occur in the gauge theory. In a transport theory with gauge invariance, the kinetic momentum is not $p_{\mu}$ but $\hat{\Pi}_{\mu}$. The energy average of the zeroth component of the second term in (4.30) yields the equal-time operator $\hat{\pi}_{0}$ given in (C2), and $\hat{\pi}_{0}=-\frac{\sqrt{3}}{2 \Lambda} \hat{f}_{01}$ in turn generates the coefficient in front of the second bracket in (4.12). In a transport theory without gauge freedom all coefficients $\hat{f}_{i, i+1}$ vanish due to the absence of linear terms in $p_{\mu}$ in the covariant constraint equation.

We have not had the patience to carry the above considerations to higher orders in the energy moments. The corresponding calculations become extremely messy. Based on the experience with pure scalar theory we expect all higher order transport equations to be redundant, but we have failed to discover a simple calculational technique which permits us to prove this in an elegant way.

\section{B. Comparison with the Feshbach-Villars approach}

In Ref. [8] a set of equal-time transport equations for scalar QED was derived by directly Wigner transforming the equations of motion for the (non-covariant) equal-time density matrix. Since the Klein-Gordon equation (4.1) contains a second-order time derivative, its direct translation into 3-dimensional phase space does not lead to a sensible transport equation which should have only first-order time derivatives. Therefore the 3-dimensional approach exploits the Feshbach-Villars representation [14 of the field equations of motion 
which contains only first-order time derivatives. The price to pay (in addition to the loss of manifest Lorentz covariance) is the introduction of an auxiliary field which results in a rather complicated $2 \times 2$ matrix structure of the scalar Wigner function. For the energy averaging method advocated here there is no such problem. In this case second-order time derivatives appear only in the constraint hierarchy, and the transport hierarchy contains only first-order time derivatives.

Expressing the equal-time density operator $\Phi(\boldsymbol{x}, \boldsymbol{y}, t)$ of the scalar field in terms of the Feshbach-Villars spinors, performing a 3-dimensional Wigner transformation and using the Klein-Gordon equation in Feshbach-Villars form, the authors of 8 ] obtained the following coupled equations for the 4 spinor components $f_{i}(\boldsymbol{x}, \boldsymbol{p}, t)(i=0,1,2,3)$ of the Wigner function:

$$
\begin{aligned}
& m \hat{d}_{t} f_{0}=-\hat{\boldsymbol{\pi}} \cdot \hat{\boldsymbol{d}}\left(f_{2}+f_{3}\right), \\
& m \hat{d}_{t} f_{1}=-\left(\frac{1}{4} \hat{\boldsymbol{d}}^{2}-\hat{\boldsymbol{\pi}}^{2}\right)\left(f_{2}+f_{3}\right)+2 m^{2} f_{2}, \\
& m \hat{d}_{t} f_{2}=\left(\frac{1}{4} \hat{\boldsymbol{d}}^{2}-\hat{\boldsymbol{\pi}}^{2}\right) f_{1}+\hat{\boldsymbol{\pi}} \cdot \hat{\boldsymbol{d}} f_{0}-2 m^{2} f_{1}, \\
& m \hat{d}_{t} f_{3}=-\left(\frac{1}{4} \hat{\boldsymbol{d}}^{2}-\hat{\boldsymbol{\pi}}^{2}\right) f_{1}-\hat{\boldsymbol{\pi}} \cdot \hat{\boldsymbol{d}} f_{0} .
\end{aligned}
$$

In Ref. [8] these equal-time phase-space distributions were related to the physical phase-space densities $\rho, \boldsymbol{j}, \epsilon$, and $\boldsymbol{P}$ as follows [8, 15]:

$$
\begin{aligned}
\rho & =e f_{0}, \\
\epsilon & =\frac{\boldsymbol{p}^{2}}{2 m}\left(f_{2}+f_{3}\right)+m f_{3}, \\
\boldsymbol{j} & =\frac{e}{m} \boldsymbol{p}\left(f_{2}+f_{3}\right), \\
\boldsymbol{P} & =\boldsymbol{p} f_{0}
\end{aligned}
$$

Unlike the spinor decomposition for spinor QED [6]:7] where each component of the Wigner function has a definite physical meaning, there is no obvious physical interpretation for the component $f_{1}$ in scalar QED.

The comparison of the charge currents $\boldsymbol{j}$ in Eqs. (4.15c) and (4.6) leads directly to the identification 


$$
W=\frac{1}{m}\left(f_{2}+f_{3}\right) .
$$

Using the relations (4.15) and (4.16) one finds from a suitable linear combination of the kinetic equations (4.14) the following equations of motion for the physical phase-space distributions $\rho$ and $\epsilon$,

$$
\begin{aligned}
& \frac{1}{e} \hat{d}_{t} \rho+\hat{\boldsymbol{\pi}} \cdot \hat{\boldsymbol{d}} W=0 \\
& \hat{d}_{t} \epsilon+\frac{1}{e} \hat{\boldsymbol{\pi}} \cdot \hat{\boldsymbol{d}} \rho-\frac{1}{2}\left(\left(\frac{\hat{\boldsymbol{d}}^{2}}{4}-\hat{\boldsymbol{\pi}}^{2}\right) \hat{d}_{t}+\hat{d}_{t} \boldsymbol{p}^{2}\right) W=0, \\
& \epsilon=\left(\frac{1}{4}\left(\hat{d}_{t}^{2}-\frac{1}{2} \hat{\boldsymbol{d}}^{2}\right)+\frac{1}{2}\left(\hat{\boldsymbol{\pi}}^{2}+\boldsymbol{p}^{2}\right)+m^{2}\right) W
\end{aligned}
$$

as well as the following relation between the unphysical component $f_{1}$ and the scalar density:

$$
f_{1}=-\frac{1}{2} \hat{d}_{t} W
$$

Comparing Eqs. (4.17) from the equal-time Feshbach-Villars approach with the corresponding Eqs. (4.7) from the covariant approach using our energy-averaging procedure, one realizes that they have a very different structure. The difference is even more obvious when looking at the corresponding equations for the space-time densities which are obtained by integrating Eqs. (4.17) over momentum:

$$
\begin{aligned}
& \partial_{t} \rho(\boldsymbol{x}, t)+\boldsymbol{\nabla} \cdot \boldsymbol{j}(\boldsymbol{x}, t)=0, \\
& \partial_{t} \epsilon(\boldsymbol{x}, t)+\boldsymbol{\nabla} \cdot \boldsymbol{P}(\boldsymbol{x}, t)-\boldsymbol{E}(\boldsymbol{x}, t) \cdot \boldsymbol{j}(\boldsymbol{x}, t)-\frac{\hbar^{2} \nabla_{x}^{2}}{8} \partial_{t} \int \frac{d^{3} p}{(2 \pi)^{3}} W(\boldsymbol{x}, \boldsymbol{p}, t)=0, \\
& \epsilon(x)=\int \frac{d^{3} p}{(2 \pi)^{3}}\left(\frac{1}{4}\left(\partial_{t}^{2}-\frac{1}{2} \boldsymbol{\nabla}_{x}^{2}\right)+\boldsymbol{p}^{2}+m^{2}\right) W(\boldsymbol{x}, \boldsymbol{p}, t) .
\end{aligned}
$$

The first equation describes charge conservation and agrees with our Eq. (4.9a). When comparing Eqs. (4.9b) and (4.19b) one sees that the energy-momentum conservation law derived from the Feshbach-Villars approach features a strange additional term of unknown physical origin. Similarly, when compared to Eq. (4.9g), Eq. (4.19d) contains a strange factor $\frac{1}{2}$ in front of the Laplace operator which breaks Lorentz invariance. We believe that these discrepancies indicate a technical problem with the Feshbach-Villars approach of 
Ref. [8]. Whether the problem resides in the derivation of the transport equations (4.14) or in the postulated relations (4.15) between the Feshbach-Villars spinor components $f_{i}$ and the physical densities could not be clarified. Only in the case of spatially constant electromagnetic fields (which to our knowledge is the only situation to which these equations have so far been applied [8, 12]) the two sets of equations (4.9) and (4.19) coincide. If we further neglect the magnetic field $\boldsymbol{B}$, we derive two decoupled ordinary differential equations in time for $W$ and $\rho$ :

$$
\begin{aligned}
& d_{t} \rho=0 \\
& \left(d_{t}^{3}+4\left(\boldsymbol{p}^{2}+m^{2}\right) d_{t}+4 e \boldsymbol{E} \cdot \boldsymbol{p}\right) W=0 .
\end{aligned}
$$

These equations are identical in both approaches. The first equation expresses charge conservation in a homogeneous electric field, and the second equation is the well-known equation

of motion for the charge current $\boldsymbol{j}$ 11, 12 which, according to the relation $\boldsymbol{j}=e \frac{p}{\sqrt{\boldsymbol{p}^{2}+m^{2}}} n$, governs the time evolution of the particle density $n$ by pair production in the electric field. This latter quantity thus comes out the same in both approaches.

\section{Spinor QED}

The case of spinor QED has been discussed in the context of the energy averaging method in Refs. [12,17,18]. The discussion presented in those papers has, however, focussed entirely on the equal-time kinetic equations for the lowest energy moment of the covariant Wigner function. Here we will reformulate the problem in terms of the equal-time hierarchy of energy moments as introduced in Sec. II and also discuss the equations of motion for the higher order moments.

Let us briefly review the relevant technical steps. We begin by performing a spinor decomposition [6] of the covariant Wigner function, separating in a second step explicitly the temporal and spatial parts [7] of the covariant spinor components:

$$
W(x, p)=\frac{1}{4}\left[F(x, p)+i \gamma_{5} P(x, p)+\gamma_{\mu} V^{\mu}(x, p)+\gamma_{\mu} \gamma_{5} A^{\mu}(x, p)+\frac{1}{2} \sigma_{\mu \nu} S^{\mu \nu}(x, p)\right]
$$




$$
\begin{aligned}
= & \frac{1}{4}\left[\gamma_{0} F_{0}(x, p)+\gamma_{5} \gamma_{0} F_{1}(x, p)+i \gamma_{5} F_{2}(x, p)+F_{3}(x, p)\right. \\
& \left.-\gamma_{5} \boldsymbol{\gamma} \cdot \boldsymbol{G}_{0}(x, p)-\boldsymbol{\gamma} \cdot \boldsymbol{G}_{1}(x, p)+i \gamma_{5} \boldsymbol{\gamma} \cdot \boldsymbol{G}_{2}(x, p)+\gamma_{5} \gamma_{0} \boldsymbol{\gamma} \cdot \boldsymbol{G}_{3}(x, p)\right] .
\end{aligned}
$$

Inserting the decomposition (4.21a) into the covariant (VGE) equation of motion [6] for the Wigner function $W(x, p)$,

$$
\left(\gamma^{\mu}\left(\hat{\Pi}_{\mu}(x, p)+\frac{i}{2} \hat{D}_{\mu}(x, p)\right)-m\right) W(x, p)=0
$$

with $\hat{\Pi}_{\mu}(x, p)$ and $\hat{D}_{\mu}(x, p)$ from Eqs. (4.3]c,d), and separating real and imaginary parts one arrives at two groups of coupled covariant kinetic equations of type (2.2), see Eqs. $(74,75)$ of Ref. [12. Performing the energy average then leads to two groups of equal-time kinetic hierarchies (with 16 such hierarchies of equations in each group) for the energy moments of the 16 covariant spinor components.

Since for spinor QED $M=0$, minimal truncation of these $2 \times 16$ hierarchies according to Sec. IIB results in one transport equation from each of the 16 transport hierarchies and no constraint equations. The minimal subgroup of equal-time kinetic equations thus consists only of the 16 transport equations for the 16 zeroth-order energy moments $f_{i}(\boldsymbol{x}, \boldsymbol{p}, t)$ and $\boldsymbol{g}_{i}(\boldsymbol{x}, \boldsymbol{p}, t)$ of the covariant spinor components $F_{i}(x, p)$ and $\boldsymbol{G}_{i}(x, p)(i=0,1,2,3)$ :

$$
\begin{aligned}
& \hat{d}_{t} f_{0}+\hat{\boldsymbol{d}} \cdot \boldsymbol{g}_{1}=0, \\
& \hat{d}_{t} f_{1}+\hat{\boldsymbol{d}} \cdot \boldsymbol{g}_{0}+2 m f_{2}=0, \\
& \hat{d}_{t} f_{2}+2 \hat{\boldsymbol{\pi}} \cdot \boldsymbol{g}_{3}-2 m f_{1}=0, \\
& \hat{d}_{t} f_{3}-2 \hat{\boldsymbol{\pi}} \cdot \boldsymbol{g}_{2}=0, \\
& \hat{d}_{t} \boldsymbol{g}_{0}+\hat{\boldsymbol{d}} f_{1}-2 \hat{\boldsymbol{\pi}} \times \boldsymbol{g}_{1}=0, \\
& \hat{d}_{t} \boldsymbol{g}_{1}+\hat{\boldsymbol{d}} f_{0}-2 \hat{\boldsymbol{\pi}} \times \boldsymbol{g}_{0}+2 m \boldsymbol{g}_{2}=0, \\
& \hat{d}_{t} \boldsymbol{g}_{2}+\hat{\boldsymbol{d}} \times \boldsymbol{g}_{3}+2 \hat{\boldsymbol{\pi}} f_{3}-2 m \boldsymbol{g}_{1}=0, \\
& \hat{d}_{t} \boldsymbol{g}_{3}-\hat{\boldsymbol{d}} \times \boldsymbol{g}_{2}-2 \hat{\boldsymbol{\pi}} f_{2}=0 .
\end{aligned}
$$

These 16 equations are identical with the BGR equations derived by Bialynicki-Birula, Gornicki and Rafelski [7] by Wigner transforming the equations of motion for the equal- 
time density matrix. They determine the dynamics of the zeroth-order energy moments. The three-dimensional dynamical operators occurring in these equations are identical with the ones arising in scalar QED and are given in Appendix Q

The first-order moments $f_{i}^{1}(x, \boldsymbol{p})$ and $\boldsymbol{g}_{i}^{1}(x, \boldsymbol{p})$ satisfy 16 transport equations derived [12] from the first energy moment of the covariant transport equations (2.2a),

$$
\begin{aligned}
& \frac{1}{\sqrt{3}} \hat{d}_{t} f_{0}^{1}+\frac{1}{\sqrt{3}} \hat{\boldsymbol{d}} \cdot \boldsymbol{g}_{1}^{1}+\hat{D} f_{0}+\boldsymbol{I} \cdot \boldsymbol{g}_{1}=0, \\
& \frac{1}{\sqrt{3}} \hat{d}_{t} f_{1}^{1}+\frac{1}{\sqrt{3}} \hat{\boldsymbol{d}} \cdot \boldsymbol{g}_{0}^{1}+\frac{2}{\sqrt{3}} m f_{2}^{1}+\hat{D} f_{1}+\boldsymbol{I} \cdot \boldsymbol{g}_{0}=0, \\
& \frac{1}{\sqrt{3}} \hat{d}_{t} f_{2}^{1}+\frac{2}{\sqrt{3}} \hat{\boldsymbol{\pi}} \cdot \boldsymbol{g}_{3}^{1}-\frac{2}{\sqrt{3}} m f_{1}^{1}+\hat{D} f_{2}+2 \hat{\boldsymbol{G}} \cdot \boldsymbol{g}_{3}=0, \\
& \frac{1}{\sqrt{3}} \hat{d}_{t} f_{3}^{1}-\frac{2}{\sqrt{3}} \hat{\boldsymbol{\pi}} \cdot \boldsymbol{g}_{2}^{1}+\hat{D} f_{3}-2 \hat{\boldsymbol{G}} \cdot \boldsymbol{g}_{2}=0, \\
& \frac{1}{\sqrt{3}} \hat{d}_{t} \boldsymbol{g}_{0}^{1}+\frac{1}{\sqrt{3}} \hat{\boldsymbol{d}} f_{1}^{1}+\frac{2}{\sqrt{3}} \hat{\boldsymbol{\pi}} \times \boldsymbol{g}_{1}^{1}+\hat{D} \boldsymbol{g}_{0}+\hat{\boldsymbol{I}} f_{1}-2 \hat{\boldsymbol{G}} \times \boldsymbol{g}_{1}=0, \\
& \frac{1}{\sqrt{3}} \hat{d}_{t} \boldsymbol{g}_{1}^{1}+\frac{1}{\sqrt{3}} \hat{\boldsymbol{d}} f_{0}^{1}-\frac{2}{\sqrt{3}} \hat{\boldsymbol{\pi}} \times \boldsymbol{g}_{0}^{1}+\frac{2}{\sqrt{3}} m \boldsymbol{g}_{2}^{1}+\hat{D} \boldsymbol{g}_{1}+\hat{\boldsymbol{I}} f_{0}-2 \hat{\boldsymbol{G}} \times \boldsymbol{g}_{0}=0, \\
& \frac{1}{\sqrt{3}} \hat{d}_{t} \boldsymbol{g}_{2}^{1}+\frac{1}{\sqrt{3}} \hat{\boldsymbol{d}} \times \boldsymbol{g}_{3}^{1}+\frac{2}{\sqrt{3}} \hat{\boldsymbol{\pi}} f_{3}^{1}-\frac{2}{\sqrt{3}} m \boldsymbol{g}_{1}^{1}+\hat{D} \boldsymbol{g}_{2}+\hat{\boldsymbol{I}} \times \boldsymbol{g}_{3}+2 \hat{\boldsymbol{G}} f_{3}=0, \\
& \frac{1}{\sqrt{3}} \hat{d}_{t} \boldsymbol{g}_{3}^{1}-\frac{1}{\sqrt{3}} \hat{\boldsymbol{d}} \times \boldsymbol{g}_{2}^{1}-\frac{2}{\sqrt{3}} \hat{\boldsymbol{\pi}} f_{2}^{1}+\hat{D} \boldsymbol{g}_{3}-\hat{\boldsymbol{I}} \times \boldsymbol{g}_{2}-2 \hat{\boldsymbol{G}} f_{2}=0,
\end{aligned}
$$

and 16 constraint equations derived [12] from the zeroth energy moment of the covariant constraint equation (2.2b):

$$
\begin{aligned}
& \frac{1}{\sqrt{3}} f_{0}^{1}=\hat{\boldsymbol{\pi}} \cdot \boldsymbol{g}_{1}-\hat{\pi}_{0} f_{0}+m f_{3} \\
& \frac{1}{\sqrt{3}} f_{1}^{1}=\hat{\boldsymbol{\pi}} \cdot \boldsymbol{g}_{0}-\hat{\pi}_{0} f_{1} \\
& \frac{1}{\sqrt{3}} f_{2}^{1}=-\frac{\hbar}{2} \hat{\boldsymbol{d}} \cdot \boldsymbol{g}_{3}-\hat{\pi}_{0} f_{2} \\
& \frac{1}{\sqrt{3}} f_{3}^{1}=\frac{\hbar}{2} \hat{\boldsymbol{d}} \cdot \boldsymbol{g}_{2}-\hat{\pi}_{0} f_{3}+m f_{0}, \\
& \frac{1}{\sqrt{3}} \boldsymbol{g}_{0}^{1}=\frac{\hbar}{2} \hat{\boldsymbol{d}} \times \boldsymbol{g}_{1}+\hat{\boldsymbol{\pi}} f_{1}-\hat{\pi}_{0} \boldsymbol{g}_{0}+m \boldsymbol{g}_{3} \\
& \frac{1}{\sqrt{3}} \boldsymbol{g}_{1}^{1}=\frac{\hbar}{2} \hat{\boldsymbol{d}} \times \boldsymbol{g}_{0}+\hat{\boldsymbol{\pi}} f_{0}-\hat{\pi}_{0} \boldsymbol{g}_{1}, \\
& \frac{1}{\sqrt{3}} \boldsymbol{g}_{2}^{1}=-\frac{\hbar}{2} \hat{\boldsymbol{d}} f_{3}+\hat{\boldsymbol{\pi}} \times \boldsymbol{g}_{3}-\hat{\pi}_{0} \boldsymbol{g}_{2}, \\
& \frac{1}{\sqrt{3}} \boldsymbol{g}_{3}^{1}=\frac{\hbar}{2} \hat{\boldsymbol{d}} f_{2}-\hat{\boldsymbol{\pi}} \times \boldsymbol{g}_{2}-\hat{\pi}_{0} \boldsymbol{g}_{3}+m \boldsymbol{g}_{0}
\end{aligned}
$$


A discussion similar to that in scalar QED reveals [18] that the transport equations (4.24) are not independent of the BGR equations (4.23) and the contraint equations (4.25). For instance, using Eqs. (4.25), the transport equation for $f_{0}^{1}$ can be expressed as an operator combination of the transport equations for $f_{0}$ and $\boldsymbol{g}_{1}$ :

$$
\hat{\pi}_{0}\left(\hat{d}_{t} f_{0}+\hat{\boldsymbol{d}} \cdot \boldsymbol{g}_{1}\right)-\hat{\boldsymbol{\pi}} \cdot\left(\hat{d}_{t} \boldsymbol{g}_{1}+\hat{\boldsymbol{d}} f_{0}-2 \hat{\boldsymbol{\pi}} \times \boldsymbol{g}_{0}+2 m \boldsymbol{g}_{2}\right)=0 .
$$

Therefore, the first-order moments are fully determined in terms of the solutions of the BGR equations (4.23) for the zeroth-order moments by solving the constraint equations (4.25).

Again, we have not been able to find a simple proof that the same is generally true for all higher order energy moments, and we stopped here. We do, however, believe that such a proof must exist [19], and that therefore all higher order energy moments can be directly computed from the solutions of the BGR transport equations by solving the constraint hierarchy.

It was shown in Ref. [12 that in the classical limit the simple algebraic relation (1.4) changes the structure of the constraints (4.25) for the first-order moments and turns them into additional constraints for the zeroth-order moments (i.e. the equal-time Wigner functions). These extra constraints reduce the number of independent zeroth-order moments from 16 in the quantum case to 4 in the classical limit. They are thus extremely important. As a result the BGR equations reduce to two decoupled Vlasov-type transport equations for the charge and spin distribution functions. In the general quantum case there are no such extra constraints on the equal-time Wigner functions [18]. One must solve all 16 coupled transport equations (4.23), but these solutions then fully determine also all higher order moments. These higher order moments have important physical meaning: the first-order moment of $F_{0}(x, p)$, for instance, describes the energy distribution in phase-space. With the help of the contraint (1.25a) it is given by

$$
\begin{aligned}
\epsilon(\boldsymbol{x}, \boldsymbol{p}, t) & =\operatorname{Tr} \int \frac{d E}{2 \pi} E \gamma_{0} \mathcal{W}(x, p)=\sqrt{\frac{2}{3}} f_{0}^{1}(\boldsymbol{x}, \boldsymbol{p}, t) \\
& =\sqrt{2}\left(m f_{3}(\boldsymbol{x}, \boldsymbol{p}, t)-\hat{\pi}_{0} f_{0}(\boldsymbol{x}, \boldsymbol{p}, t)+\hat{\boldsymbol{\pi}} \cdot \boldsymbol{g}_{1}(\boldsymbol{x}, \boldsymbol{p}, t)\right) .
\end{aligned}
$$




\section{CONCLUSIONS}

We have presented a universal method for the construction of equal-time quantum transport theories from the covariant quantum field equations of motion. It is based on energy averaging the covariant kinetic equations for the covariant Wigner operator (which is the Wigner transform of the covariant, "two-time" density matrix) and its energy moments. This procedure yields a hierarchy of coupled transport and constraint equations for the energy moments of the covariant Wigner function, the so-called equal-time Wigner functions. We showed how, in the mean-field approximation, this hierarchy can be truncated at a rather low level, requiring the solution of only a small number of equal-time transport equations, and how the higher order energy moments (higher order equal-time Wigner functions) can be constructed from these solutions via constraints.

The major advantage of the equal-time formulation of (quantum) transport theory is that the resulting transport equations can be solved as initial value problems, with boundary values for the equal-time Wigner functions at $t=-\infty$ which can be calculated from the fields at $t=-\infty$. This is not the case for the covariant transport equations and the covariant Wigner function. The present paper thus provides an essential step in the direction of practical computations of the dynamics of relativistic quantum field systems out of thermal equilibrium in the language of transport theory, i.e. in a phase-space oriented approach. The method presented here improves upon previous approaches by being much more systematic: we did not just focus on the lowest energy moments (which contain only a small fraction of the information contained in the covariant Wigner function), but we discussed and showed how to solve the complete hierarchy of moment equations. We had already before demonstrated for spinor QED that the non-covariant three-dimensional approach (which starts directly from the equal-time density matrix) yields an incomplete set of equal-time transport equations. In this paper we also discussed the case of scalar QED and again discovered serious problems with the direct three-dimensional approach based on the Feshbach-Villars formulation. We conclude that the only safe way of deriving a correct and 
complete set of equal-time quantum transport equations is by starting from the covariant formulation and taking energy moments of the covariant kinetic equations. The method can be generalized in a straightforward way to other types of interactions [16 [18], including non-Abelian gauge interactions [19].

The structure of the hierarchy of equal-time quantum kinetic equations depends on the structure of the covariant field equations from which one starts. For scalar or vector theories with second order time derivatives one has to solve a coupled set of three equations for the three lowest energy moments, two resulting from the equal-time transport hierarchy and one stemming from the constraint hierarchy. For spinor theories with only first order time derivatives in the field equations one ends up with only one equal-time transport equation for the lowest energy moment of each spinor component of the Wigner function. All higher order energy moments can be determined from the solutions of these equations by solving constraints.

Important further simplifications occur in the classical limit $\hbar \rightarrow 0$ : then all higher order energy moments can be expressed algebraically in terms of the zeroth energy moment, and the number of equations is drastically reduced. For scalar theories one obtains just one Vlasov-type equation for the on-shell charge distribution function. For spinor theories one obtains two decoupled Vlasov-type equations (one scalar and one vector equation) for the on-shell charge and spin density distributions in phase-space. Again the only systematic way of deriving the constraints leading to these simplifications is by energy averaging the (classical limit of the) covariant transport equations.

All results in this paper were derived in the mean field approximation, i.e. in the collisionless limit. It is generally known that including collision terms in the covariant transport equations leads to the appearance of non-localities in time ("memory effects") in the equaltime transport equations [20]. It is not inconceivable that these memory effects lead to serious complications for the truncation of the equal-time transport hierarchy. This is certainly an interesting and difficult problem for future studies. 


\section{ACKNOWLEDGMENTS}

P.Z. thanks GSI for a fellowship. The work of U.H. was supported by BMBF, DFG, and GSI. He would like to express his thanks to CERN, where this work was completed, for warm hospitality and a stimulating environment.

\section{APPENDIX A: THE COEFFICIENTS $c_{i j}^{m n}$}

¿From the definition of the coefficients $c_{i j}^{m n}$ in 2.18) and the orthogonality condition (2.5) we see that

$$
c_{i j}^{00}=\delta_{i j}
$$

Furthermore, it is easy to see from (2.18) that

$$
c_{i j}^{m n}=0 \quad \text { for } \quad n>i+m \quad \text { or } \quad j>i+m-n .
$$

Therefore, if the finite sum over $m$ has the upper limit $M$, the sum over $j$ in Eq. (2.16) extends only over the range $j \leq i+M$.

¿From the recursion relation for the Legendre polynomials

$$
\omega P_{i}(\omega)=\frac{i+1}{2 i+1} P_{i+1}(\omega)+\frac{i}{2 i+1} P_{i-1}(\omega)
$$

one easily derives the recursion relation

$$
c_{i, j}^{m+1, n}=\frac{i+1}{\sqrt{(2 i+1)(2 i+3)}} c_{i+1, j}^{m, n}+\frac{i}{\sqrt{(2 i+1)(2 i-1)}} c_{i-1, j}^{m, n}
$$

for the coefficients $c_{i j}^{m n}$. This allows to raise the first upper index $m$, starting from (A1). In particular we have

$$
\begin{aligned}
c_{i-1, i}^{10} & =\frac{i}{\sqrt{(2 i-1)(2 i+1)}}, \\
c_{i-1, i+1}^{20} & =\frac{i(i+1)}{(2 i+1) \sqrt{(2 i-1)(2 i+3)}},
\end{aligned}
$$


which we will need in Appendix B. Similarly the second upper index can be raised by using the relations

$$
\partial_{\omega}\left(P_{j+1}(\omega)-P_{j-1}(\omega)\right)=(2 j+1) P_{j}(\omega), \quad P_{j}( \pm 1)=( \pm 1)^{j} \quad(j \geq 0)
$$

which, for $j \geq 2$, lead to

$$
c_{i, j}^{m, n+1}=\sqrt{\frac{2 j+1}{2 j-3}} c_{i, j-2}^{m, n+1}+\sqrt{(2 j+1)(2 j-1)} c_{i, j-1}^{m, n} .
$$

This expression is useless for $j=0$ and $j=1$; these cases can be treated by another recursion relation which can be obtained by using (A6) on the Legendre polynomial with the index $i$ :

$$
c_{i, j}^{m, n+1}=\sqrt{\frac{2 i+1}{2 i-3}} c_{i-2, j}^{m, n+1}-\sqrt{(2 i+1)(2 i-1)} c_{i-1, j}^{m, n}-m\left(c_{i, j}^{m-1, n}-\sqrt{\frac{2 i+1}{2 i-3}} c_{i-2, j}^{m-1, n}\right) .
$$

For $i<2$ this must be used together with

$$
c_{-i, j}^{m, n}=\sqrt{-1} c_{i-1, j}^{m, n}
$$

which follows from $P_{-i}(x)=P_{i-1}(x)$.

The above recursion relations can be initialized with the following nonvanishing coefficients for $i, j, m, n \leq 1$ :

$$
c_{00}^{00}=c_{11}^{00}=-c_{00}^{11}=1, \quad c_{01}^{10}=c_{10}^{10}=\frac{1}{\sqrt{3}}, \quad c_{10}^{01}=-\sqrt{3}, \quad c_{11}^{11}=-2 .
$$

\section{APPENDIX B: THE OPERATORS $\hat{g}_{i j}$ AND $\hat{f}_{i j}$ FOR SCALAR THEORIES}

The three-dimensional dynamical operators $\hat{g}_{i j}(x, \boldsymbol{p})$ and $\hat{f}_{i j}(x, \boldsymbol{p})$ needed in Eqs. (3.14)

and (3.19) are obtained from the definition (2.17) with the coefficients $c_{i j}^{m n}$ from Appendix A. Please note that for $\hat{g}_{i j}$ the upper limit in (2.17) for the sum over $m$ is $M=1$ while for $\hat{f}_{i j}$ it is $M+1=2$. One finds:

$$
\begin{aligned}
& \hat{g}_{00}=\hat{G}_{00}-\hat{G}_{11}, \\
& \hat{g}_{01}=\frac{1}{\sqrt{3}} \hat{G}_{10},
\end{aligned}
$$




$$
\begin{aligned}
& \hat{g}_{10}=\sqrt{3}\left(-\hat{G}_{01}+\frac{1}{3} \hat{G}_{10}+2 \hat{G}_{12}\right), \\
& \hat{g}_{11}=\hat{G}_{00}-2 \hat{G}_{11}, \\
& \hat{g}_{12}=\frac{2}{\sqrt{15}} \hat{G}_{10}, \\
& \hat{g}_{20}=\sqrt{5}\left(3 \hat{G}_{02}-\hat{G}_{11}-9 \hat{G}_{13}\right), \\
& \hat{g}_{21}=\sqrt{15}\left(-\hat{G}_{01}+\frac{2}{15} \hat{G}_{10}+3 \hat{G}_{12}\right), \\
& \hat{g}_{22}=\hat{G}_{00}-3 \hat{G}_{11}, \\
& \hat{g}_{23}=\frac{3}{\sqrt{35}} \hat{G}_{10}, \\
& \hat{f}_{00}=\hat{F}_{00}-\hat{F}_{11}+\frac{1}{3} \hat{F}_{20}+2 \hat{F}_{22}, \\
& \hat{f}_{01}=\frac{1}{\sqrt{3}}\left(\hat{F}_{10}-2 \hat{F}_{21}\right), \\
& \hat{f}_{02}=\frac{2}{3 \sqrt{5}} \hat{F}_{20}, \\
& \hat{f}_{13}=\frac{2 \sqrt{3}}{5 \sqrt{7}} \hat{F}_{20} \cdot \\
& \hat{f}_{10}=\frac{2}{\sqrt{3}}\left(-\hat{F}_{01}+\frac{1}{3} \hat{F}_{10}+2 \hat{F}_{12}-\hat{F}_{21}-6 \hat{F}_{23}\right), \\
& \hat{f}_{11}=\hat{F}_{00}-2 \hat{F}_{11}+\frac{3}{5} \hat{F}_{20}+6 \hat{F}_{22}, \\
& \hat{f}_{12}= \\
& =
\end{aligned}
$$

The dynamical operators $\hat{G}_{m n}$ and $\hat{F}_{m n}$ are given in equations (3.12) for scalar mean field interactions and in Appendix 9 for scalar QED. Please note that in both cases $\hat{F}_{2 k}=0$ for $k \neq 0$ and thus some of the expressions for $\hat{f}_{i j}$ above simplify.

We also note that in

$$
\hat{f}_{i-1, i+1}=\sum_{m=0}^{2} \sum_{n=0}^{i-1+m} c_{i-1, i+1}^{m n} \hat{F}_{m n}
$$

the only term which doesn't violate the second inequality in (A2) is the one with $m=2, n=$ 0 :

$$
\hat{f}_{i-1, i+1}=c_{i-1, i+1}^{20} \hat{F}_{20}=\frac{i(i+1)}{(2 i+1) \sqrt{(2 i-1)(2 i+3)}} \hat{F}_{20}
$$


In the last equality we used (A5b). From Eqs. (3.12b) or ( 4 ( $4 \mathrm{~g}$ ) we thus see that $\hat{f}_{i-1, i+1}$ is just a constant. Following the same reasoning one also obtains

$$
\hat{g}_{i-1, i}=\sum_{m=0}^{1} \sum_{n=0}^{i+m} c_{i-1, i}^{m n} \hat{G}_{m n}=c_{i-1, i}^{10} \hat{G}_{10}=\frac{i}{\sqrt{(2 i-1)(2 i+1)}} \hat{G}_{10}
$$

where according to Eqs. (3.12a) or (C3d) $\hat{G}_{10}$ is proportional to the time derivative $\partial_{t}$ resp. $\hat{d}_{t}$

\section{APPENDIX C: THE OPERATORS $\hat{G}_{m n}$ AND $\hat{F}_{m n}$ FOR SCALAR QED}

The elementary operators $\hat{\Pi}_{\mu}$ and $\hat{D}_{\mu}$ are extensions of the covariant momentum $p_{\mu}$ and the covariant derivative $\partial_{\mu}$, respectively. Their double expansions in $\omega$ and $\partial / \partial \omega$ are

$$
\begin{aligned}
& \hat{\Pi}_{0}=\Lambda \omega+\hat{\pi}_{0}+\frac{\hat{A}}{\Lambda} \frac{\partial}{\partial \omega}-\frac{\hat{B}}{\Lambda^{2}} \frac{\partial^{2}}{\partial \omega^{2}}-\frac{\hat{C}}{\Lambda^{3}} \frac{\partial^{3}}{\partial \omega^{3}}+\cdots \\
& \hat{D}_{0}=\hat{d}_{t}-\frac{\hat{D}}{\Lambda} \frac{\partial}{\partial \omega}-\frac{\hat{E}}{\Lambda^{2}} \frac{\partial^{2}}{\partial \omega^{2}}+\frac{\hat{F}}{\Lambda^{3}} \frac{\partial^{3}}{\partial \omega^{3}}+\cdots \\
& \hat{\boldsymbol{\Pi}}=\hat{\boldsymbol{\pi}}-\frac{\hat{\boldsymbol{G}}}{\Lambda} \frac{\partial}{\partial \omega}+\frac{\hat{\boldsymbol{H}}}{\Lambda^{2}} \frac{\partial^{2}}{\partial \omega^{2}}+\cdots \\
& \hat{\boldsymbol{D}}=-\hat{\boldsymbol{d}}+\frac{\hat{\boldsymbol{I}}}{\Lambda} \frac{\partial}{\partial \omega}+\frac{\hat{\boldsymbol{J}}}{\Lambda^{2}} \frac{\partial^{2}}{\partial \omega^{2}}+\cdots
\end{aligned}
$$

with the equal-time dynamical operators,

$$
\begin{aligned}
& \hat{\pi}_{0}(x, \boldsymbol{p})=i e \hbar \int_{-\frac{1}{2}}^{\frac{1}{2}} d s s \boldsymbol{E}\left(\boldsymbol{x}+i s \hbar \boldsymbol{\nabla}_{p}, t\right) \cdot \boldsymbol{\nabla}_{p}, \\
& \hat{\boldsymbol{\pi}}(x, \boldsymbol{p})=\boldsymbol{p}-i e \hbar \int_{-\frac{1}{2}}^{\frac{1}{2}} d s s \boldsymbol{B}\left(\boldsymbol{x}+i s \hbar \boldsymbol{\nabla}_{p}, t\right) \times \boldsymbol{\nabla}_{p}, \\
& \hat{d}_{t}(x, \boldsymbol{p})=\hbar \partial_{t}+e \hbar \int_{-\frac{1}{2}}^{\frac{1}{2}} d s \boldsymbol{E}\left(\boldsymbol{x}+i s \hbar \boldsymbol{\nabla}_{p}, t\right) \cdot \boldsymbol{\nabla}_{p}, \\
& \hat{\boldsymbol{d}}(x, \boldsymbol{p})=\hbar \boldsymbol{\nabla}_{x}+e \hbar \int_{-\frac{1}{2}}^{\frac{1}{2}} d s \boldsymbol{B}\left(\boldsymbol{x}+i s \hbar \boldsymbol{\nabla}_{p}, t\right) \times \boldsymbol{\nabla}_{p}, \\
& \hat{A}(x, \boldsymbol{p})=e \hbar^{2} \int_{-\frac{1}{2}}^{\frac{1}{2}} d s s^{2} \frac{\partial}{\partial t} \boldsymbol{E}\left(\boldsymbol{x}+i s \hbar \boldsymbol{\nabla}_{p}, t\right) \cdot \boldsymbol{\nabla}_{p}, \\
& \hat{B}(x, \boldsymbol{p})=\frac{i e \hbar^{3}}{2} \int_{-\frac{1}{2}}^{\frac{1}{2}} d s s^{3} \frac{\partial^{2}}{\partial t^{2}} \boldsymbol{E}\left(\boldsymbol{x}+i s \hbar \boldsymbol{\nabla}_{p}, t\right) \cdot \boldsymbol{\nabla}_{p}, \\
& \hat{C}(x, \boldsymbol{p})=\frac{e \hbar^{4}}{6} \int_{-\frac{1}{2}}^{\frac{1}{2}} d s s^{4} \frac{\partial^{3}}{\partial t^{3}} \boldsymbol{E}\left(\boldsymbol{x}+i s \hbar \boldsymbol{\nabla}_{p}, t\right) \cdot \boldsymbol{\nabla}_{p},
\end{aligned}
$$




$$
\begin{aligned}
\hat{D}(x, \boldsymbol{p})= & i e \hbar^{2} \int_{-\frac{1}{2}}^{\frac{1}{2}} d s s \frac{\partial}{\partial t} \boldsymbol{E}\left(\boldsymbol{x}+i s \hbar \boldsymbol{\nabla}_{p}, t\right) \cdot \boldsymbol{\nabla}_{p} \\
\hat{E}(x, \boldsymbol{p})= & \frac{e \hbar^{3}}{2} \int_{-\frac{1}{2}}^{\frac{1}{2}} d s s^{2} \frac{\partial^{2}}{\partial t^{2}} \boldsymbol{E}\left(\boldsymbol{x}+i s \hbar \boldsymbol{\nabla}_{p}, t\right) \cdot \boldsymbol{\nabla}_{p} \\
\hat{F}(x, \boldsymbol{p})= & \frac{i e \hbar^{4}}{6} \int_{-\frac{1}{2}}^{\frac{1}{2}} d s s^{3} \frac{\partial^{3}}{\partial t^{3}} \boldsymbol{E}\left(\boldsymbol{x}+i s \hbar \boldsymbol{\nabla}_{p}, t\right) \cdot \boldsymbol{\nabla}_{p} \\
\hat{\boldsymbol{G}}(x, \boldsymbol{p})= & e \hbar^{2} \int_{-\frac{1}{2}}^{\frac{1}{2}} d s s^{2} \frac{\partial}{\partial t} \boldsymbol{B}\left(\boldsymbol{x}+i s \hbar \boldsymbol{\nabla}_{p}, t\right) \times \boldsymbol{\nabla}_{p}+i e \hbar \int_{-\frac{1}{2}}^{\frac{1}{2}} d s s \boldsymbol{E}\left(\boldsymbol{x}+i s \hbar \boldsymbol{\nabla}_{p}, t\right), \\
\hat{\boldsymbol{H}}(x, \boldsymbol{p})= & \frac{i e \hbar^{3}}{2} \int_{-\frac{1}{2}}^{\frac{1}{2}} d s s^{3} \frac{\partial^{2}}{\partial t^{2}} \boldsymbol{B}\left(\boldsymbol{x}+i s \hbar \boldsymbol{\nabla}_{p}, t\right) \times \boldsymbol{\nabla}_{p} \\
& -e \hbar^{2} \int_{-\frac{1}{2}}^{\frac{1}{2}} d s s^{2} \frac{\partial}{\partial t} \boldsymbol{E}\left(\boldsymbol{x}+i s \hbar \boldsymbol{\nabla}_{p}, t\right), \\
\hat{\boldsymbol{I}}(x, \boldsymbol{p})= & i e \hbar^{2} \int_{-\frac{1}{2}}^{\frac{1}{2}} d s s \frac{\partial}{\partial t} \boldsymbol{B}\left(\boldsymbol{x}+i s \hbar \boldsymbol{\nabla}_{p}, t\right) \times \boldsymbol{\nabla}_{p}-e \hbar \int_{-\frac{1}{2}}^{\frac{1}{2}} d s \boldsymbol{E}\left(\boldsymbol{x}+i s \hbar \boldsymbol{\nabla}_{p}, t\right), \\
\hat{\boldsymbol{J}}(x, \boldsymbol{p})= & \frac{e \hbar^{3}}{2} \int_{-\frac{1}{2}}^{\frac{1}{2}} d s s^{2} \frac{\partial^{2}}{\partial t^{2}} \boldsymbol{B}\left(\boldsymbol{x}+i s \hbar \boldsymbol{\nabla}_{p}, t\right) \times \boldsymbol{\nabla}_{p} \\
& +i e \hbar^{2} \int_{-\frac{1}{2}}^{\frac{1}{2}} d s s \frac{\partial}{\partial t} \boldsymbol{E}\left(\boldsymbol{x}+i s \hbar \boldsymbol{\nabla}_{p}, t\right) .
\end{aligned}
$$

By substituting these expansions into the expressions of $\hat{G}$ and $\hat{F}$ in (4.3) and using the definition of $\hat{G}_{m n}(2.9)$, we list some low-order $\hat{G}_{m n}$ and $\hat{F}_{m n}$ which will be used in the derivation of the kinetic equations for low-order energy moments,

$$
\begin{aligned}
& \hat{G}_{00}=\hat{\pi}_{0} \hat{d}_{t}+\hat{\boldsymbol{\pi}} \cdot \hat{\boldsymbol{d}} \\
& \hat{G}_{01}=-\frac{1}{\Lambda}\left(\hat{\pi}_{0} \hat{D}+\hat{\boldsymbol{\pi}} \cdot \hat{\boldsymbol{I}}-\hat{A} \hat{d}_{t}+\hat{\boldsymbol{G}} \cdot \hat{\boldsymbol{d}}\right) \\
& \hat{G}_{02}=\frac{1}{\Lambda^{2}}\left(\hat{\boldsymbol{H}} \cdot \hat{\boldsymbol{d}}+\hat{\boldsymbol{G}} \cdot \hat{\boldsymbol{I}}-\hat{\boldsymbol{\pi}} \cdot \hat{\boldsymbol{J}}-\hat{B} \hat{d}_{t}-\hat{A} \hat{D}-\hat{\pi}_{0} \hat{E}\right) \\
& \hat{G}_{10}=\Lambda \hat{d}_{t} \\
& \hat{G}_{11}=-\hat{D} \\
& \hat{G}_{12}=-\frac{\hat{E}}{\Lambda} \\
& \hat{G}_{13}=\frac{\hat{F}}{\Lambda^{2}}
\end{aligned}
$$

and

$$
\hat{F}_{00}=\frac{1}{4}\left(\hat{d}_{t}^{2}-\hat{\boldsymbol{d}}^{2}\right)-\left(\hat{A}+\hat{\pi}_{0}^{2}-\hat{\boldsymbol{\pi}}^{2}\right)+m^{2}
$$




$$
\begin{aligned}
& \hat{F}_{01}=\frac{1}{\Lambda}\left(\frac{1}{4}\left(\{\hat{\boldsymbol{d}}, \hat{\boldsymbol{I}}\}-\left\{\hat{d}_{t}, \hat{D}\right\}\right)-2 \hat{\pi}_{0} \hat{A}-\{\hat{\boldsymbol{\pi}}, \hat{\boldsymbol{G}}\}+2 \hat{B}\right), \\
& \hat{F}_{02}=\frac{1}{\Lambda^{2}}\left(\frac{1}{4}\left(\hat{D}^{2}-\hat{\boldsymbol{I}}^{2}+\{\hat{\boldsymbol{d}}, \hat{\boldsymbol{J}}\}-\left\{\hat{d}_{t}, \hat{E}\right\}\right)-\left(\hat{A}^{2}-\hat{\boldsymbol{G}}^{2}-\{\hat{\boldsymbol{\pi}}, \hat{\boldsymbol{H}}\}-2 \hat{\pi}_{0} \hat{B}\right)+3 \hat{C}\right), \\
& \hat{F}_{10}=-2 \Lambda \hat{\pi}_{0}, \\
& \hat{F}_{11}=-2 \hat{A} \\
& \hat{F}_{12}=2 \frac{\hat{B}}{\Lambda} \\
& \hat{F}_{20}=-\Lambda^{2} .
\end{aligned}
$$




\section{REFERENCES}

* $\quad$ On leave from Hua-Zhong Normal University, Wuhan, China.

[1] H.-Th. Elze and U. Heinz, Phys. Rep. 183, 81 (1989).

[2] P. Carruthers and F. Zachariasen, Rev. Mod. Phys. 55, 245 (1983).

[3] U. Heinz, Phys. Rev. Lett. 51, 351 (1983).

[4] H.-Th. Elze, M. Gyulassy and D. Vasak, Nucl. Phys. B276, 706 (1986).

[5] H.-Th. Elze, M. Gyulassy and D. Vasak, Phys. Lett.B177, 402 (1986).

[6] D. Vasak, M. Gyulassy and H.-Th. Elze, Ann. Phys. (N.Y.) 173, 462 (1987).

[7] I. Bialynicki-Birula, P. Gornicki and J. Rafelski, Phys. Rev. D44, 1825 (1991).

[8] C. Best, P. Gornicki and W. Greiner, Ann. Phys. (N.Y.) 225, 169(1993).

[9] P. Henning, Phys. Rep. 253, 263 (1995).

[10] J. Schwinger, Phys. Rev. 82, 664 (1951);

Y. Kluger, J. M. Eisenberg, B. Svetitsky, F. Cooper and E. Mottola, Phys. Rev. Lett. 67, 2427 (1991); and Phys. Rev. D45, 4659 (1992).

[11] C. Best and J. M. Eisenberg, Phys. Rev. D47, 4639 (1993).

[12] P. Zhuang and U. Heinz, Ann. Phys. (N.Y.) 245, 311 (1996);

and ibid., Erratum 249, 362 (1996).

[13] S. R. deGroot, W. A. van Leeuwen, and Ch. G. van Weert, "Relativistic Kinetic Theory", NorthHolland, Amsterdam, 1980.

[14] H. Feshbach and F. Villars, Rev. Mod. Phys. 30, 24 (1958).

[15] The last equation (4.15d) was given incorrectly in [8] and must be corrected as shown (C. Best, private communication). 
[16] G. R. Shin and J. Rafelski, Ann. Phys. (N. Y.) 243, 65 (1995).

[17] P. Zhuang and U. Heinz, Phys. Rev. D53, 2096 (1996).

[18] A. Abada, M. C. Birse, P. Zhuang and U. Heinz, Phys. Rev. D54, 4175 (1996).

[19] We just learned that S. Ochs has apparently found such a general proof (S. Ochs, PhD thesis, University of Regensburg, Dec. 1996, unpublished).

[20] R. Malfliet, in Correlations and Clustering Phenomena in Subatomic Physics, edited by M.N. Harakeh, O. Scholten, and J.H. Koch, NATO ASI Series (Plenum, New York, 1996), in press. 